\title{
Microscopic Pore Structure of Surrounding Rock for Underground Strategic Petroleum Reserve (SPR) Caverns in Bedded Rock Salt
}

\author{
Nan Zhang ${ }^{1, *}$, Wei Liu ${ }^{2, *}$, Yun Zhang ${ }^{1}$, Pengfei Shan ${ }^{1} \mathbb{D}$ and Xilin Shi ${ }^{3}$ \\ 1 Key Laboratory of Western Mines and Hazard Prevention, Ministry of Education of China, School of Energy \\ Science and Engineering, Xi'an University of Science and Technology, Xi'an 710054, China; \\ zhangyun@cumt.edu.cn (Y.Z.); shanpengfei@xust.edu.cn (P.S.) \\ 2 State Key Laboratory of Coal Mine Disaster and Control, Chongqing University, Chongqing 400044, China \\ 3 State Key Laboratory of Geomechanics and Geotechnical Engineering, Institute of Rock and Soil Mechanics, \\ Chinese Academy of Sciences, Wuhan 430071, China; xlshi@whrsm.ac.cn \\ * Correspondence: zhangnan0032@foxmail.com (N.Z.); whrsmliuwei@126.com (W.L.)
}

Received: 4 February 2020; Accepted: 24 March 2020; Published: 27 March 2020

\begin{abstract}
Using salt caverns for an underground strategic petroleum reserve (SPR) is considered as an ideal approach due to the excellent characteristics of low porosity, low permeability, self-healing of damage, and strong plastic deformation ability of rock salt. Salt deposits in China are mostly layered rock salt structures, with the characteristics of many interlayers, bringing great challenges for the construction of SPR facilities. Studying the microscopic pore characteristics of the rock surrounding SPR salt caverns in different environments (with brine and crude oil erosion) is necessary because the essence of mechanical and permeability characteristics is the macroscopic embodiment of the microscopic pore structure. In this paper, XRD tests and SEM tests are carried out to determine the physical properties of storage media and surrounding rock. Gas adsorption tests and mercury intrusion tests are carried out to analyze the microscopic pore structure, specific surface area variation and total aperture distribution characteristics of SPR salt cavern host rock. Results show that: (1) Large numbers of cores in interlayer and caprock may provide favorable channels for the leakage of high-pressure crude oil and brine. (2) The blockage of pores by macromolecular organic matter (colloid and asphaltene) in crude oil will not significantly change the structural characteristics of the rock skeleton, which is beneficial to the long-term operation of the SPR salt cavern. (3) The water-rock interaction will bring obvious changes in the micro-pore structure of rock and increase the leakage risk of the storage medium. The results can provide theoretical bases and methods for the tightness analysis of China's first underground SPR salt cavern.
\end{abstract}

Keywords: strategic petroleum reserve (SPR); bedded rock salt; salt cavern; pore characteristic; gas adsorption

\section{Introduction}

The dynamic development of the world economy means that the demand for energy is increasing from year to year in virtually all sectors of this economy [1,2]. Energy is closely related to economy, environment, and social development [3-5]. Especially with the fast development of the economy and the resulting rapid growth of energy consumption [6-13], the need for energy is increasingly growing, especially for fossil energy such as crude oil, leading to the increasing dependence on foreign oil every year [14]. In 2018, China's oil demand exceeded 600 million tons for the first time, and its dependence on foreign countries was close to $70 \%$. The statistics of China's total oil consumption and dependence on foreign countries from 2003 to 2017 are shown in Figure 1. According to international 
energy security standards, a country's dependence on foreign oil is "unsafe" in the range of $40 \%-50 \%$, and more than $50 \%$ is "in crisis" [15]. Since 2008, China's dependence on foreign oil has exceeded $50 \%$, and currently it is close to $70 \%$. This shows that oil safety in China is already facing severe tests. Ensuring the country's oil safety is always a major strategic issue. Therefore, it is urgent to establish a large-scale strategic petroleum reserve system.

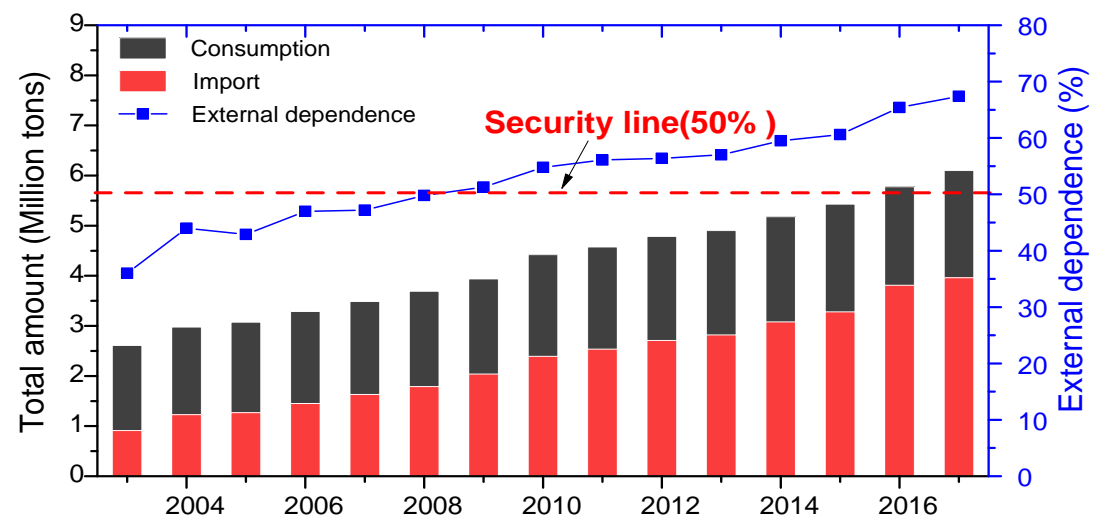

Figure 1. The current reserve and security situation of crude oil in China.

Rock salt is considered as an ideal medium for crude oil storage due to its excellent characteristics of low porosity, low permeability, self-healing of damage, and strong plastic deformation ability [16-19]. Nearly 36 countries have implemented underground oil storage, with $90 \%$ of the oil in the United States, 50\% in Germany and 30\% in France are stored in rock salt caverns [20]. However, there is no engineering practice of constructing salt cavern for oil storage in China. According to the second and third phases of the national strategic petroleum reserve plan, China's petroleum reserve will gradually shift from the surface ground to the underground, with more strategic depth and safety than in the first phase. The reserve mode will also shift from the traditional surface ground storage tank to granite chamber with water seal, salt cavern storage and other modes. The construction of salt caverns such as Jintan in Jiangsu, Huai'an, and Yunying in Hubei are all under this plan [21]. To date, the construction of underground salt cavern oil storage in China is still in the stage of demonstration, and the underground salt cavern oil storage is far behind that of some developed countries. However, with the advancement of the national strategic petroleum reserve plan, the implementation of underground petroleum reserves in rock salt formations will become the key deployment direction of future strategic petroleum reserves. Carrying out the necessary research and engineering work, such as the pilot test and the evaluation of the stability and tightness of the SPR salt caverns, which could provide a theoretical and experimental basis for the construction of China's first salt cavern oil storage, is urgent.

Salt deposits in China are mostly layered rock salt structures, and their geological occurrence conditions are extremely complex, with many rock salt layers, thin single-layer thickness, and many insolubles and insoluble interlayers [19,22-24]. The interlayers seriously affect the sealing characteristics of surrounding rocks of the SPR salt caverns, which are also the key to determine whether salt caverns can store crude oil safely and reliably [25]. Once the salt cavern leaks, it will lead to oil leakage, which may bring the risk of serious disasters such as cavity scrapping and ground subsidence, even causing major fires and activating regional faults [26-28]. Therefore, the porosity and permeability characteristics of the rock surrounding the caverns are important for evaluating its tightness [29-32]. Moreover, the essence of these macroscopic characteristics is the macroscopic embodiment of its microscopic pore structure. The microstructure of rock often has a strong control of its macro mechanics and seepage behavior. An in-depth study of the microscopic pore structure characteristics of reservoir surrounding rock has guiding significance for understanding its macroscopic characteristics and engineering application. 
Many research studies have been conducted to analyze the porosity and permeability characteristics of bedded rock salt. Nan Zhang et al. [33] analyzed the mechanical and permeability properties of mudstone interlayers around the SPR salt cavern in bedded rock salt. Jianwen Chen et al. [34] investigated the microscopic pore structures of rock salt. They found that the microscopic pore structure of rock salt plays a dominant role in its permeability. Cuevas et al. [35] used three independent methods to analyze the characteristic of the pore space of rock salt, showing that infraporosity consists of a bundle of non-intersecting pores, whereas microporosity can be considered as a network of pores and throats. Li et al. [36] proposed a three-dimensional expanded Cosserat medium constitutive model for bedded rock salt and use it in a stability analysis of underground salt caverns. Shi et al. [37] studied the role of tensile strength development of the mudstone interlayer subject to a brine immersion environment. Liu et al. [38] studied the physical and mechanical properties as well as the porosity and permeability of bedded rock salt through laboratory tests and show that the bedded rock salt is satisfactory for the tightness and stability of caverns. Yang et al. [39] discovered that the relationship between the permeability and porosity of mudstones is a key constitutive equation for modeling subsurface fluid flow and is fundamental to the quantification of a range of geological processes. Ji et al. [40] analyzed the relationship between porosity and permeability, pore structure and distribution characteristics of the salt caverns. All this research provides a solid foundation for the first underground SPR salt cavern construction in China.

The rocks surrounding oil storage salt caverns are in contact with different storage media under different operation stages [41]. These contacts are mainly divided into the following three categories: (1) long-term contact between surrounding rock and brine in the cavity before crude oil injection but after completion of cavity formation; (2) the long-term contact between crude oil and surrounding rocks in the long-term storage state after the oil filling of the oil storage cavern has been completed; (3) brine (freshwater) contacts surrounding rock again after crude oil is replaced by brine during the oil extraction stage. Actually, the SPR salt cavern is finished by injecting water and removing the brine. Similarly, oil is removed by displacing it with water injection. As is shown in Figure 2, during the cavern leaching stage and the oil drainage stage, the surrounding rocks are eroded by high-pressure brine. However, during the storage operation, the surrounding rocks are eroded by high-pressure crude oil. The properties of mudstone interlayers in contact with oil or brine are not clear. What is more, these properties are the most crucial factor to ensure the SPR salt cavern long-term safe and stable operation, due to the essence of the mechanical and permeability characteristics of the surrounding rock being the macroscopic embodiment of its microscopic pore structure. Therefore, it is necessary to measure the physical properties and study the micro-characteristics of the surrounding rock of salt cavern oil storage in different environments. Meanwhile, different storage media will have different effects on the characteristics of the rock surrounding the reserve. At present, very little work has been done on related studies of SPR in bedded rock salt.

In this paper, a microscopic pore structure analysis is presented of SPR salt caverns in bedded rock salt in Jintan, China. The structure of this paper is as follows: in Sections 2 and 3, the experimental scheme and the composition analysis tests of storage media and SEM analysis of SPR cavern surrounding rocks are described. In Section 4, the pore structure characteristics of cavern surrounding rocks and their specific surface area variation characteristics are described. In Section 5, the total aperture distribution characteristics of cavern-surrounding rocks are described. In Sections 6 and 7, some discussions and conclusions are presented. This will lay a foundation for further study on the macro-mechanical characteristics and porosity and permeability characteristics of surrounding rock of SPR salt cavern of Jintan, as well as a basic reference for similar engineering practices in other places. 


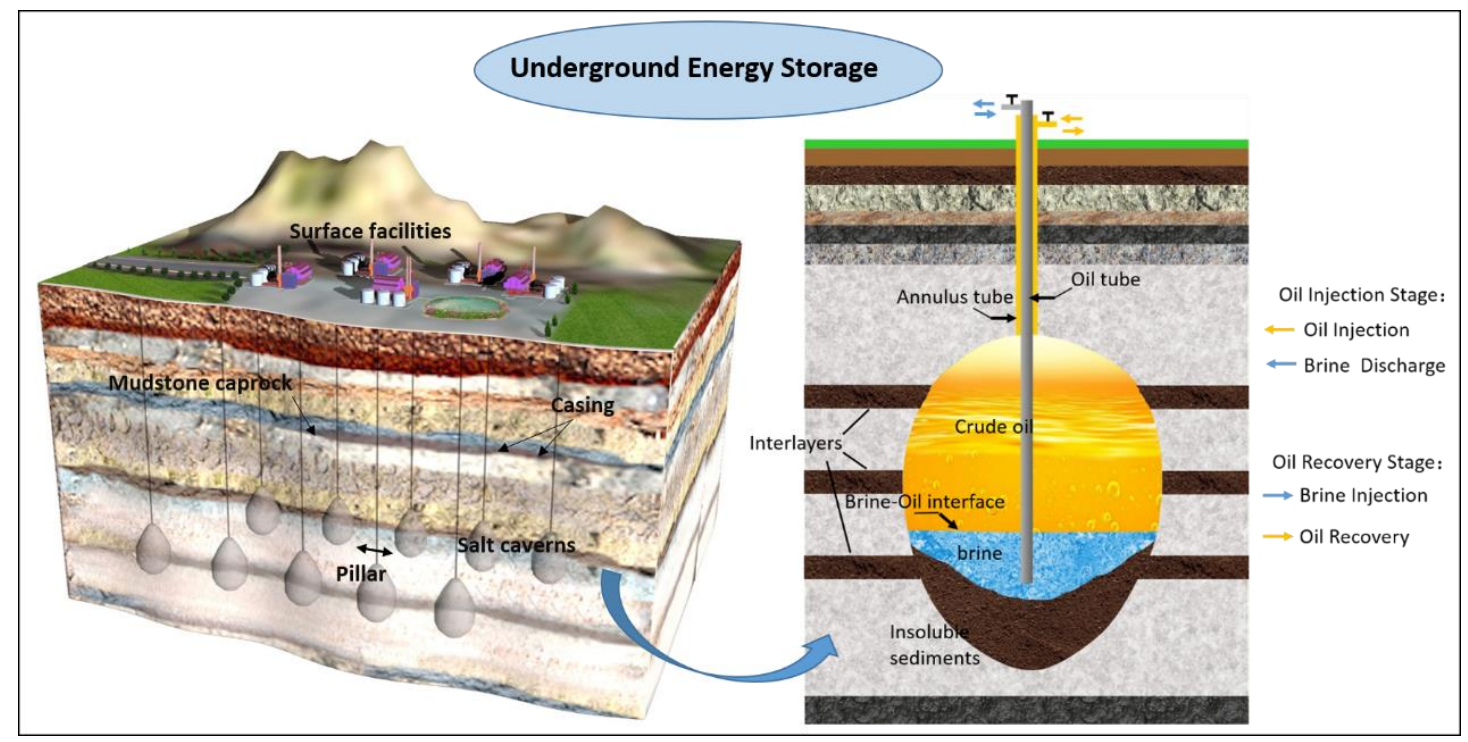

Figure 2. The process of crude oil injection-production of SPR in rock salt.

\section{Physical Properties}

\subsection{Physical Properties of Storage Media}

\subsubsection{Crude Oil Composition}

The petroleum in the national strategic petroleum reserve plan generally refers to crude oil that has not been processed. It is usually a dark brown mixture of liquid hydrocarbons such as alkanes, cycloalkanes, aromatic hydrocarbons, and olefins. Its condensation point is between $-50 \sim 35^{\circ} \mathrm{C}$. The relative density is generally between $0.75 \times 10^{3}$ and $0.97 \times 10^{3} \mathrm{~kg} / \mathrm{m}^{3}$. Colloids and asphaltenes are the basic components of crude oil. The crude oil used in all the tests in this paper was obtained from Jianghan Oilfield of Sinopec. The basic physical parameters of the crude oil were measured in Jianghan Oilfield Exploration and Development Research Institute. The results are shown in Table 1.

Table 1. Basic physical parameters of crude oil.

\begin{tabular}{|c|c|c|c|c|c|c|c|}
\hline $\begin{array}{c}\text { Sample } \\
\text { NO. }\end{array}$ & $\begin{array}{l}\text { Density } \\
\left(\mathrm{kg} / \mathrm{m}^{3}\right)\end{array}$ & $\begin{array}{c}\text { Dynamic } \\
\text { Viscosity } \\
(\mathrm{mPa} \cdot \mathrm{s})\end{array}$ & $\begin{array}{l}\text { Condensation } \\
\text { Point }\left({ }^{\circ} \mathrm{C}\right)\end{array}$ & Water (\%) & Wax $(\%)$ & $\begin{array}{l}\text { Colloids } \\
\text { (\%) }\end{array}$ & $\begin{array}{c}\text { Asphaltene } \\
(\%)\end{array}$ \\
\hline$Y-1$ & $0.85 \times 10^{3}$ & 4.134 & 26 & 0.99 & 7.9 & 11.8 & 1.75 \\
\hline$Y-2$ & $0.86 \times 10^{3}$ & 4.765 & 27 & 1.02 & 9.2 & 10.1 & 1.96 \\
\hline $\mathrm{Y}-3$ & $0.84 \times 10^{3}$ & 4.865 & 25 & 1.02 & 8.1 & 9.3 & 1.81 \\
\hline AVG. & $0.85 \times 10^{3}$ & 4.588 & 26 & 1.01 & 8.4 & 10.4 & 1.84 \\
\hline
\end{tabular}

\subsubsection{Brine Composition}

The brine used in the test was obtained from the brine collection site of Jintan Salt Mine. The brine samples were divided into four groups (of which sample S- 4 is the saturated brine sample of the salt mine). The chemical composition of the samples was analyzed. The analysis results are shown in Table 2. The results show that the main ions in the brine samples are $\mathrm{Na}^{+}$and $\mathrm{Cl}^{-}$, containing a small amount of $\mathrm{SO}_{4}{ }^{2-}, \mathrm{Ca}^{2+}$ and a very small amount of other ions such as $\mathrm{Mg}^{2+}$, $\mathrm{K}^{+}$, etc. 
Table 2. Component analysis of brine samples of Jintan salt mine.

\begin{tabular}{cccccccc}
\hline Sample & Density $(\mathrm{g} / \mathrm{mL})$ & $\mathbf{N a}^{+}(\mathrm{g} / \mathrm{L})$ & $\mathrm{Cl}^{-}(\mathrm{g} / \mathrm{L})$ & $\mathbf{S O}_{4}{ }^{2+}(\mathrm{g} / \mathrm{L})$ & $\mathbf{C a}^{2+}(\mathrm{g} / \mathrm{L})$ & $\mathbf{M g}^{2+}(\mathbf{m g} / \mathrm{L})$ & $\mathbf{K}^{+}(\mathbf{m g} / \mathrm{L})$ \\
\hline S-1 & 1.141 & 81.16 & 124.7 & 5.35 & 1.09 & 8.40 & 17.22 \\
S-2 & 1.172 & 100.30 & 156.0 & 6.79 & 1.04 & 8.08 & 20.06 \\
S-3 & 1.192 & 116.80 & 181.7 & 6.89 & 1.14 & 8.74 & 20.86 \\
S-4 & 1.202 & 118.20 & 187.3 & 4.63 & 0.79 & 12.30 & 22.16 \\
\hline
\end{tabular}

\subsection{Physical Properties of Rocks Surrounding the Cavern}

\subsubsection{XRD Analysis}

The samples of the surrounding rock are taken from the depth ranging from 840-1100 m of well M17 in Jintan salt mine. That is because the proposed depth of the SPR salt caverns range from 960-1060 m. Therefore, rock samples collected in this depth range will have better representativeness. The mineral composition of the rock was determined by the Bruker AXS D8-Focus X-ray diffractometer. The mineral compositions of rock salt, interlayer and cap rock are shown in Tables 3-5.

Table 3. Mineral components of rock salt determined by XRD.

\begin{tabular}{cccccccc}
\hline \multirow{2}{*}{ Sample } & \multicolumn{7}{c}{ Mineral Component (\%) } \\
\cline { 2 - 7 } & NaCl & Quartz & Analcite & Dolomite & Ankerite & Glauberite & Clay Minerals \\
\hline YY-01 & 90.70 & 3.18 & 0.58 & 1.82 & - & - & 3.72 \\
YY-02 & 94.05 & 2.55 & - & 2.86 & 0.54 & - & - \\
YY-03 & 91.48 & 1.26 & 0.28 & 2.64 & - & 0.35 & 3.99 \\
YY-04 & 92.29 & 1.85 & 0.69 & 1.15 & 0.68 & 0.48 & 2.86 \\
AVG. & 92.13 & 2.21 & 0.39 & 2.12 & 0.31 & 0.21 & 2.64 \\
\hline
\end{tabular}

Table 4. Mineral composition of interlayer determined by XRD.

\begin{tabular}{cccccccccccc}
\hline \multirow{2}{*}{ Sample } & \multicolumn{10}{c}{ Mineral Component (\%) } \\
\cline { 2 - 11 } & Albite & Analcite & Quartz & Pyrite & Spilite & Feldspar & Ankerite & NaCl & Calcite & Glauberite & Clay Minerals \\
\hline JC-01 & 8.64 & 8.93 & 13.66 & 7.24 & 4.37 & 6.91 & 12.27 & 5.23 & 8.41 & 10.88 \\
JC-02 & 6.31 & 11.23 & 16.23 & 3.54 & 5.68 & 8.24 & 18.10 & 2.01 & 0.82 & 11.24 & 13.46 \\
JC-03 & 9.63 & 9.24 & 11.75 & 2.39 & 2.87 & 7.60 & 17.63 & 6.88 & 11.58 & 4.16 & 16.60 \\
JC-04 & 11.53 & 8.46 & 14.32 & 8.25 & 2.71 & 8.79 & 10.75 & 4.72 & 5.73 & 5.42 \\
AVG. & 9.03 & 9.47 & 13.99 & 5.36 & 3.91 & 7.89 & 14.69 & 4.71 & 6.64 & 7.93 & 19.32 \\
\hline
\end{tabular}

Table 5. Mineral components of cap rock determined by XRD.

\begin{tabular}{ccccccccc}
\hline \multirow{2}{*}{ Sample } & \multicolumn{7}{c}{ Mineral Component (\%) } \\
\cline { 2 - 8 } & Quartz & NaCl & Analcite & Feldspar & Albite & Pyrite & Calcite & Clay Minerals \\
\hline GC-01 & 41.43 & 2.83 & 8.27 & 11.33 & 9.87 & 1.13 & 4.04 & 21.10 \\
GC-02 & 38.59 & 3.65 & 5.26 & 4.96 & 16.33 & 6.22 & 4.87 & 20.12 \\
GC-03 & 28.33 & 2.82 & 6.81 & 7.12 & 10.95 & 7.23 & 7.35 & 29.39 \\
GC-04 & 36.29 & 1.84 & 5.68 & 5.75 & 9.85 & 8.62 & 9.45 & 22.52 \\
AVG. & 36.16 & 2.76 & 6.51 & 7.29 & 11.75 & 5.80 & 6.43 & 23.28 \\
\hline
\end{tabular}

According to XRD test results, the average content of sodium chloride in rock salt samples is $92.13 \%$. The main mineral components of the interlayer are albite, analcite, quartz, pyrite, spilite, feldspar, ankerite, rock salt, calcite, glauberite, and clay minerals. Quartz, albite and clay minerals account for $71.19 \%$ of the total mineral composition of the cap rock sample. This will play a decisive effect on the physical and mechanical properties of the cap rock. 


\subsubsection{Scanning Electron Microscope (SEM) Analysis}

The physical and mechanical properties of surrounding rock and its porosity and permeability depend on its mineral composition and skeleton structure composed of mineral particles. In order to analyze the microstructure of rock salt, interlayers and cap rock in SPR salt caverns, an SEM characterization was carried out. It can recognize the size, occurrence, and distribution of minerals, pore shape of rocks, throat size, the structure of particle epidermis and pore throat wall, etc. It can also identify the blockage of pore throats after contact with the fluid.

Scanning results of rock samples are shown in Figure 3. It can be seen that the surface of the rock salt sample is extremely compact. The crystal grains are large and the cementation between the crystal grains is extremely tight. After being magnified 2000 times, only a few pores on the surface of the crystal grains can be seen, which shows that the pores of the rock salt are far smaller than the micron level, while the pores larger than the micron level obtained through other pore size analysis experiments are more likely to be caused by micro-cracks in the rock salt sample. This reflects the essential reason for the extremely low permeability and porosity of rock salt. Mineral particles in interlayer samples are small and mostly in the form of clusters or sheets, with many surface pores and uneven pore size distribution. The cap layer samples have two distinct pore structures: crystal structure and lamellar structure. Compared with rock salt, the number of micropores in interlayer and caprock may provide favorable channels for the leakage of high-pressure crude oil and brine.

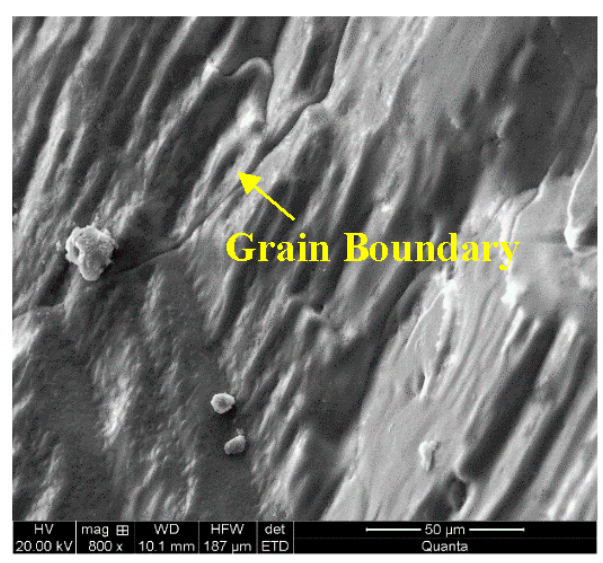

(a) Rock salt, magnified 800 times

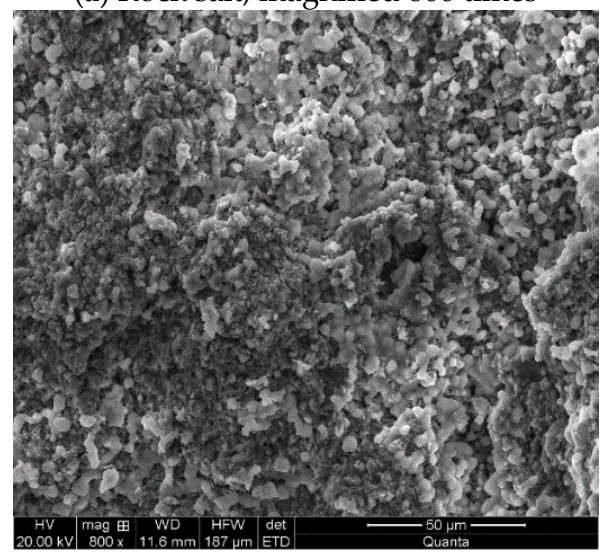

(c) Interlayers, magnified 800 times

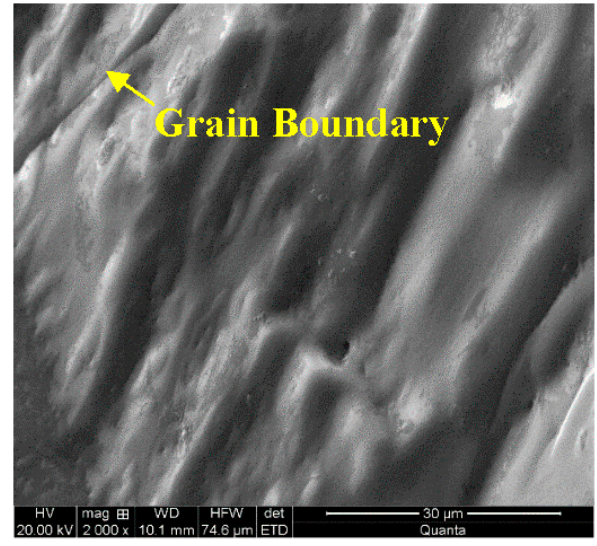

(b) Rock salt, magnified 2000 times

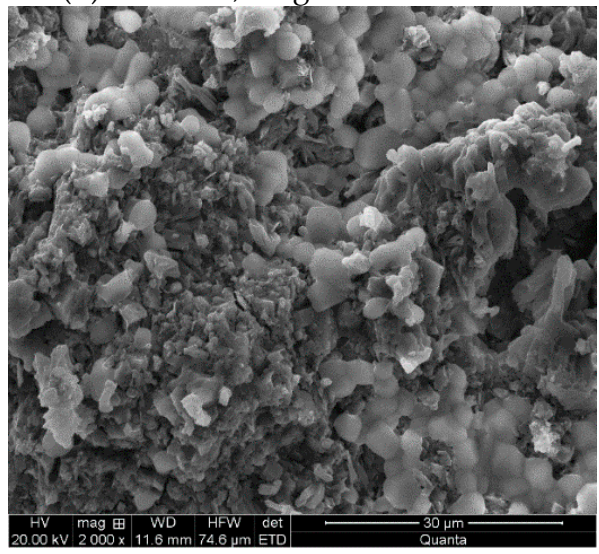

(d) Interlayers, magnified 2000 times

Figure 3. Cont. 


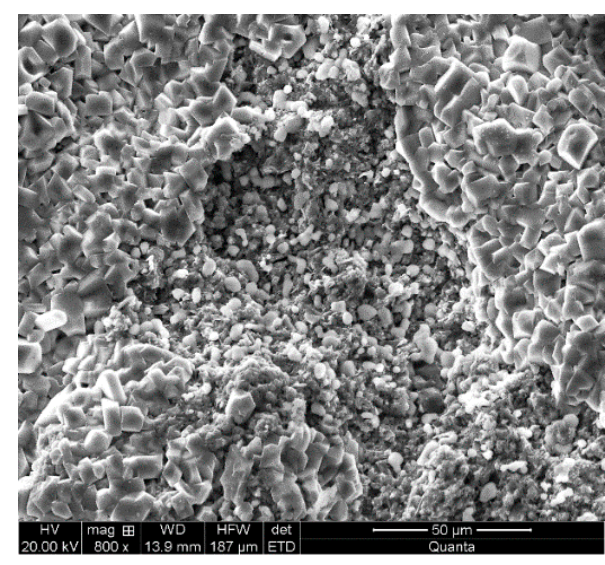

(e) Cap rock, magnified 800 times

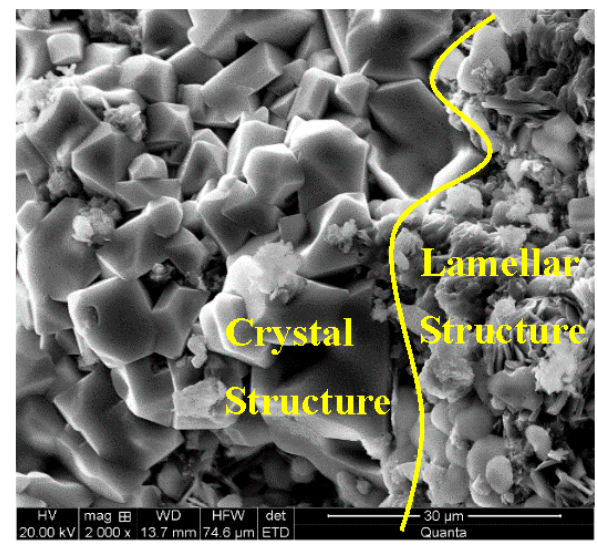

(f) Cap rock, magnified 2000 times

Figure 3. Electron microscope scans of the rock surrounding the strategic petroleum reserve (SPR) salt cavern.

\section{Experimental Preprocessing Condition and Equipment}

To study the pore structure characteristics of cavern surrounding rocks under crude oil immersion and brine immersion conditions, our research group independently designed a liquid phase immersion test device for the tests, as is shown in Figure 4. It can simulate the underground environment in the SPR caverns based on the in-situ stress and temperature data measured in the field. The pressure of the device ranges from 0 to $50 \mathrm{MPa}$, and its accuracy is $0.1 \mathrm{MPa}$. The control temperature of the device ranges from 0 to $110^{\circ} \mathrm{C}$, and its accuracy is $0.1^{\circ} \mathrm{C}$.

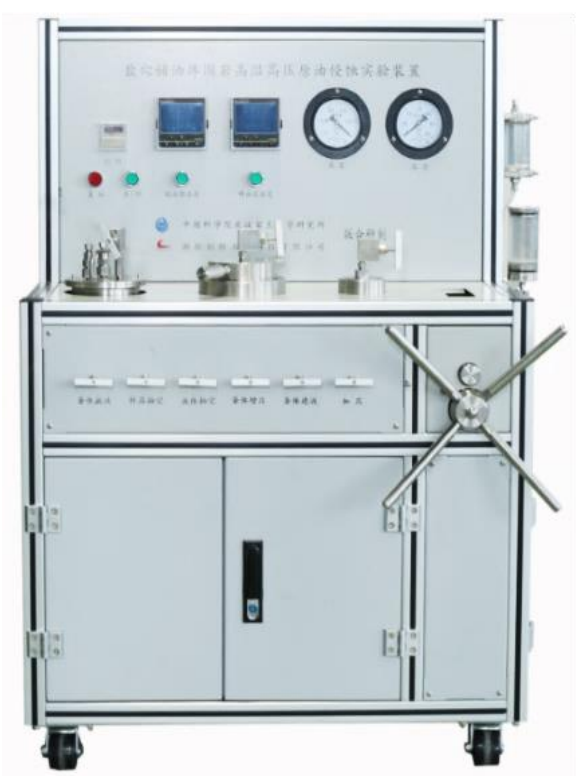

Figure 4. The immersion test device.

The temperature in this test was set at $50{ }^{\circ} \mathrm{C}$ according to the measured brine temperature at $1000 \mathrm{~m}$ depth. The pressure was set as the hydraulic pressure of the oil or brine applied to the mudstone interlayer at $1000 \mathrm{~m}$ depth. The hydraulic pressure is calculated as $\mathrm{P}=\mathrm{P}_{\mathrm{w}}+\gamma_{\text {liquid }} \mathrm{h}$, where $\mathrm{P}_{\mathrm{w}}$ is the wellhead pressure; $\gamma_{\text {liquid }}$ is the unit weight of brine or crude oil; $h$ is the calculated depth. According to the previous research, the hydraulic pressure of the brine on the surrounding rock is about $12 \mathrm{MPa}$ $\left(\gamma_{\text {water }}=12 \mathrm{kPa} / \mathrm{m}, \mathrm{P}_{\mathrm{w}}=0 \mathrm{MPa}\right)$. The hydraulic pressure of the crude oil on the surrounding rock is 
about $14 \mathrm{MPa}\left(\gamma_{\mathrm{oil}}=8.20 \mathrm{kPa} / \mathrm{m}, \mathrm{P}_{\mathrm{w}}=6 \mathrm{MPa}\right)$. The hydraulic pressure schematic graph of the SPR salt cavern are shown in Figure 5.

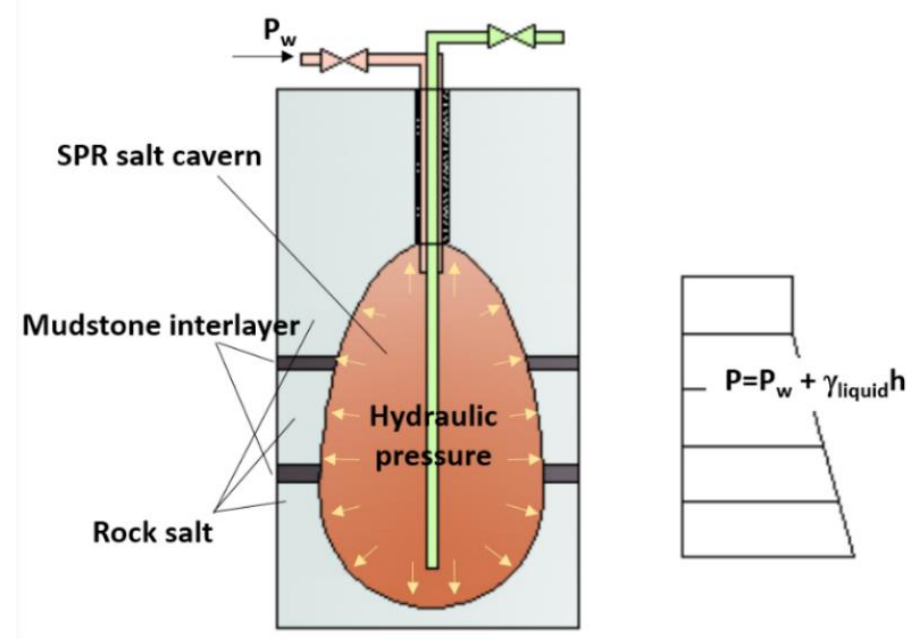

Figure 5. The hydraulic pressure schematic graph of the SPR salt cavern.

All of the gas adsorption test samples and mercury injection test samples in Sections 4 and 5 were preprocessed by the immersion test device before testing.

\section{Pore Structure Characteristics of Cavern Surrounding Rocks}

The gas adsorption method can effectively analyze the pore structure of porous media and reflect the pore distribution of micropores and mesopores [34]. This method has been widely recognized and applied in the measurement of the pore structure of porous media. In this section, the pore structure characteristics and specific surface area changes in rock surrounding a salt cavern under the four different conditions of natural state, crude oil erosion, brine erosion and washing oil (control) are studied through static nitrogen adsorption tests. The influence of different media storage conditions on the pore structure of the rock is discussed.

\subsection{Test Condition}

A static nitrogen adsorption test was completed in the testing center of the Wuhan Institute of Geotechnical Mechanics, Chinese Academy of Sciences. Before the test, in order to eliminate the residual bound water and capillary water in the samples, all samples are vacuumed at $300{ }^{\circ} \mathrm{C}$ for $3 \mathrm{~h}$. In the experiment, high purity nitrogen was used as the adsorbate, and the nitrogen adsorption capacity at different relative pressures was measured in a liquid nitrogen $77.35 \mathrm{~K}$ low-temperature environment. The adsorption isotherm is plotted with the relative pressure as the abscissa and the nitrogen adsorption amount as the ordinate.

According to the erosion of surrounding rock by different media during the construction and operation of the SPR salt cavern, the preparation of rock salt, interlayer and cap rock samples is divided into three different conditions: the natural environment, brine erosion and crude oil erosion. After that, static nitrogen adsorption tests are carried out. For rock salt samples we did not consider the brine erosion condition because of their water solubility. In order to characterize the influence of organic matter residue on surrounding rock pores, the samples saturated with crude oil are divided into two situations as control: crude oil erosion and oil washing (the samples eroded by crude oil are put into organic solvent for $24 \mathrm{~h}$ oscillation cleaning), considering that some organic matter in crude oil will remain after high temperature. Basic information about test samples and test conditions is shown in Table 6. 
Table 6. Basic information about test samples and test conditions.

\begin{tabular}{cccc}
\hline Sample NO. & Depth/m & Lithology & Condition \\
\hline $1-1$ & & & Natural environment \\
$1-2$ & $979.61-979.83$ & Rock salt & $\begin{array}{c}\text { Crude oil erosion } \\
\text { Oil washing }\end{array}$ \\
\hline $1-3$ & & & $\begin{array}{c}\text { Natural environment } \\
\text { Brine erosion }\end{array}$ \\
$2-1$ & & Interlayer & Crude oil erosion \\
$2-3$ & $923.58-923.72$ & & Oil washing \\
$2-4$ & & & Natural environment \\
$3-1$ & & Brine erosion \\
$3-2$ & & Cap rock & Crude oil erosion \\
$3-3$ & $875.23-875.55$ & & Oil washing \\
\hline $3-4$ & & & \\
\hline
\end{tabular}

\subsection{Adsorption Isotherm Characteristics}

\subsubsection{Pore Structure of Rock Salt under Different Conditions}

Under natural conditions, the adsorption isotherm of rock salt sample 1-1 is shown in Figure 6a. The adsorption isotherm has a sudden upward trend when the relative pressure $\mathrm{P} / \mathrm{P}_{0}$ is in the range of $0-0.03$. It shows that all samples contain a certain proportion of micropores and micropore filling occurs. When the relative pressure reaches about 0.5 , the adsorbate begins to show capillary condensation, and the adsorption isotherm rises rapidly. The adsorption saturation trend appears at a higher relative pressure when the adsorption isotherm begins to flatten, a type IV adsorption isotherm, (defined by IUPAC) [42]. This indicates that the surface of the rock salt has small pores, medium pores, and large pores, and the large pores do not exceed the measurement range of static nitrogen adsorption, and its pore diameter is less than $500 \mathrm{~nm}$.

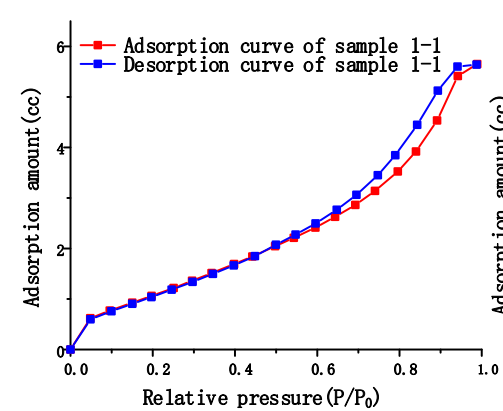

(a) Natural condition

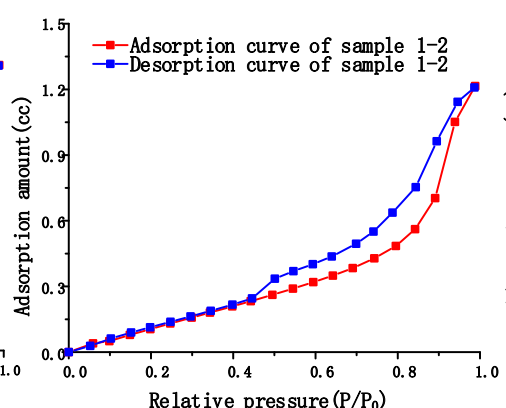

(b) Crude oil erosion

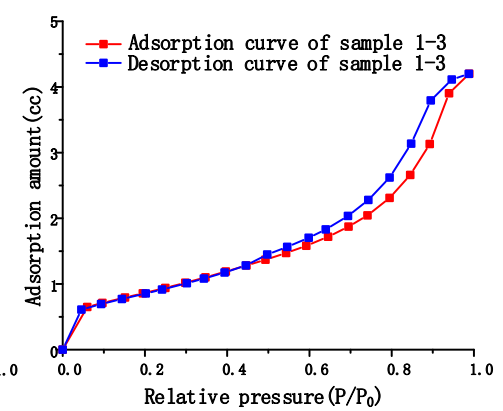

(c) Oil washing

Figure 6. The adsorption isotherms of rock salt samples under different sample treatment conditions.

The shape of the adsorption loop clearly reflects the pore structure of rock salt samples. Comparing the classification of adsorption loops in adsorption isotherms, it can be seen that the adsorption and desorption curves in sample 1-1 are nearly parallel, belonging to class A adsorption loops [43]. This indicates that the pore structure of rock salt consists of tubular capillary pores with both ends open.

The adsorption isotherm of the sample under the condition of crude oil erosion is shown in Figure $6 \mathrm{~b}$. The adsorption isotherm morphology of sample 1-2 is slightly different from that of sample 1-1, but still belongs to type IV adsorption isotherm. The adsorption loop is still a Class A loop. This shows that the pore structure of rock salt does not change with crude oil erosion. However, when the relative pressure is low, its adsorption isotherm is different from the one for natural conditions. When the relative pressure $P / P_{0}$ is in the range of $0-0.03$, the micropore filling phenomenon observed in sample 1-1 disappears, indicating that the number of micropores number in sample 1-2 decreases under the crude oil erosion. When the relative pressure is close to saturation, the amount of nitrogen 
adsorption of sample $1-2$ is $1.21 \mathrm{cc}$, which is only $21.4 \%$ of the total adsorption amount of $5.65 \mathrm{cc}$ of sample 1-1 under natural conditions.

When the crude oil in the sample is shaken and cleaned by an organic solvent, we find that the adsorption isotherm and adsorption loop morphology of samples 1-3 are basically the same as that of rock salt sample 1-1 under natural conditions, as shown in Figure $6 c$. When the relative pressure $P / P_{0}$ is in the range of $0-0.03$, the micropore pore filling phenomenon appears again. After washing the oil, the adsorption amount of nitrogen rises to $4.21 \mathrm{cc}$, which is $74.5 \%$ of the total adsorption amount under natural conditions. This shows that, as the residual organic matter in the pores is washed away, the pores of the rock salt gradually return to the structure close to the natural conditions, indicating that the erosion of crude oil does not significantly change the pore size distribution and pore structure morphological characteristics of the rock salt. It is presumed that the disappearance of micropores in sample 1-2 is caused by the filling and covering of micropores in rock salt pores by the residual organic matter in the crude oil.

\subsubsection{Pore Structure of Interlayer under Different Conditions}

The adsorption isotherms of interlayer samples under different conditions are shown in Figure 7a-d. The adsorption isotherms are basically similar in shape and all of them are of reverse " $S$ " type, all belonging to type II adsorption isotherms. When the relative pressure $P / P_{0}$ is smaller, in the range of $0-0.03$, the adsorption isotherms of the samples under other conditions all rise abruptly, like those of the rock salt samples, except for sample 2-3 under the condition of crude oil erosion. This indicates that the interlayer sample also contains a certain proportion of micropores, and micropore filling occurs. However, under the condition of crude oil erosion, the adsorption isotherm of sample 2-3 has an upward trend, but it is a relatively slow one. It is presumed that although the organic matter in crude oil still covers and fills some micropores in the interlayer, some micropores still remain, resulting in micropore filling, even if it is not obvious. However, as the relative pressure increases gradually, the adsorption capacity also increases, indicating the existence of mesopores. The sharp rise in the adsorption isotherm in the second half shows that, with the increase in multi-layer adsorption layers, capillary condensation begins to occur in the pores, and there is no saturated adsorption trend until the saturated vapor pressure is close. This means that the pore size range of the interlayer samples ranges from small to large until it is out of the range of the measuring instrument. Therefore, when it is necessary to measure the total pore size distribution of the interlayer, the pore size distribution measured by static nitrogen adsorption is incomplete. Thus, the mercury intrusion method should be combined to measure the distribution of macropores so as to obtain the complete chart of the total pore size distribution of the interlayer.

The shape of the adsorption loop still clearly reflects the pore structure of the interlayer samples. Compared with the adsorption loops in adsorption isotherms, interlayer samples under different conditions all belong to Class B adsorption loops, indicating that the pore structure of both brine erosion and crude oil erosion is consistent with that of the interlayer under natural conditions. All of them suggest the presence of slit-like capillary pores with parallel walls. This shows that brine and crude oil erosion have not changed the basic morphological characteristics of the pore structure of the interlayer, but it should be emphasized that this does not mean that there is no influence on the interlayer under the brine and crude oil erosion. The adsorption capacity of samples with the same mass is obviously different under different conditions. The adsorption capacity of sample 2-1 under natural conditions was the largest, reaching $50.72 \mathrm{cc}$. The adsorption capacity of sample 2-2 under brine erosion condition decreased to $42.12 \mathrm{cc}$, which was $16.96 \%$ lower than under natural conditions, while the adsorption capacity of sample 2-3 under crude oil erosion was the lowest, $25.54 \mathrm{cc}$, which was $49.65 \%$ lower than that under natural conditions. After washing with oil, the adsorption capacity of sample 2-4 rose to $44.11 \mathrm{cc}$, which was $86.97 \%$ of the adsorption capacity of sample $2-1$. This means that the adsorption capacity of interlayer samples is different under different conditions. This is closely related to the specific surface area of the samples under various conditions. Since adsorption occurs on 
the surface of the adsorbent, the specific surface area of the adsorbent is an important factor affecting the adsorption capacity. The larger the specific surface area, the stronger the adsorption capacity. Therefore, it is very important to study the change characteristics of the specific surface area of samples under different conditions to explore the influence of crude oil and brine on pore characteristics.

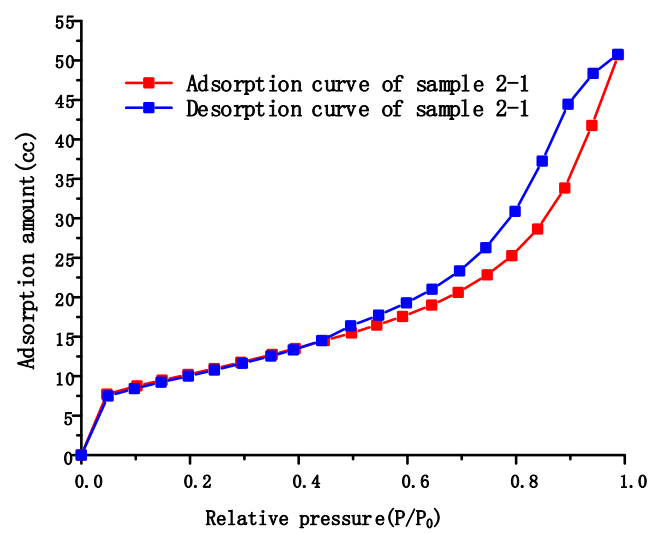

(a) Natural condition

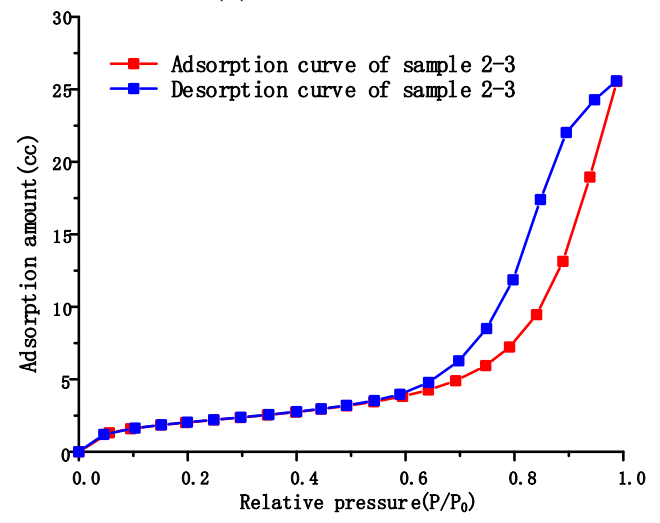

(c) Crude oil erosion

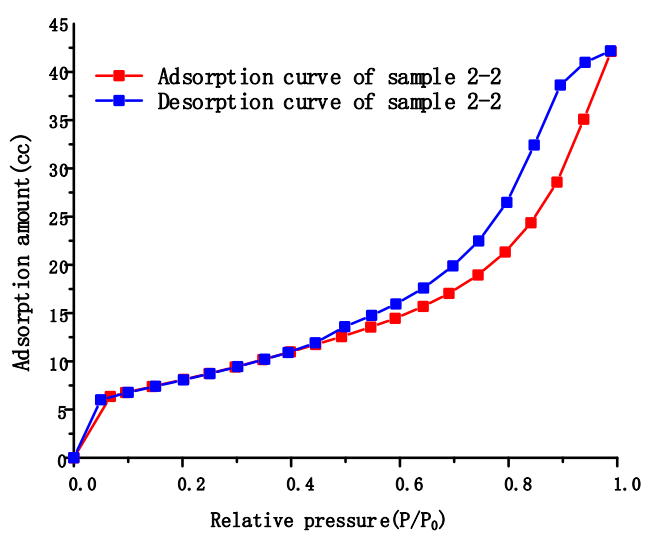

(b) Brine erosion

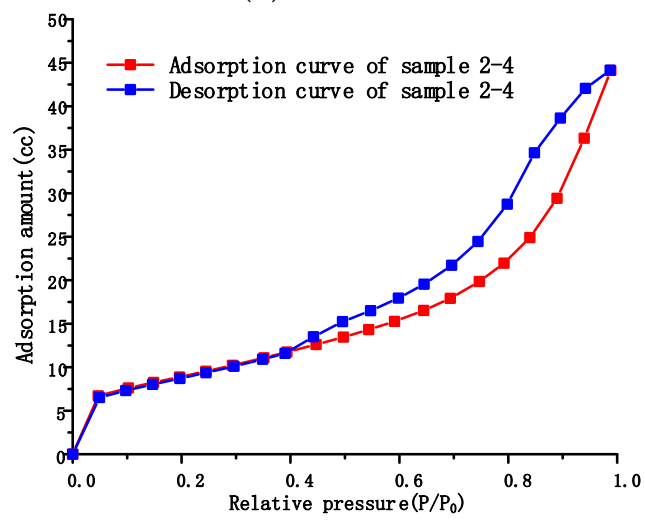

(d) Oil washing

Figure 7. The adsorption isotherms of interlayer samples under different conditions.

\subsubsection{Pore Structure of Caprock under Different Conditions}

The adsorption isotherms of cover samples under different conditions are shown in Figure 8a-d. Similar to the adsorption isotherms of interlayer samples, the adsorption isotherms of caprock samples all belong to Class II adsorption isotherms. Micropore filling also occurs when the relative pressure is low, and capillary condensation occurs when the relative pressure is close to saturation. This shows that the pores of the caprock samples also consist of small pores, mesopores and macropores, and the pore size distribution of the macropores still needs further determination by mercury intrusion. The adsorption amounts of the samples under four different conditions are as follows: natural condition sample 3-1 $(28.32 \mathrm{cc})>$ washed oil sample 3-4 $(24.86 \mathrm{cc})>$ brine erosion sample 3-2 $(22.20 \mathrm{cc})>$ crude oil erosion sample 3-3 $(3.21 \mathrm{cc})$. This rule is consistent with interlayer samples under different conditions, and the causes of this phenomenon will be discussed in the next section.

The cap-rock samples under different conditions all present atypical adsorption loops, which do not belong to any of the five types of adsorption loops from A to E, but are the superposition of several typical loops. According to the comparison of adsorption loops, it is found that the cap-rock samples can be considered to be formed by the superposition of class A adsorption loops and class D adsorption loops, which may be due to the pore structure of the cap layer not only having tubular capillary pores with both ends open, but also having tapered capillary pores with all sides open. This means that it not only has tubular structures but also lamellar structures. This kind of adsorption isotherm morphology 
is often related to the high content of clay in the crystal grains of the material, which is verified by the scanning electron microscope observation results of the cap layer, as shown in Figure $3 \mathrm{f}$.

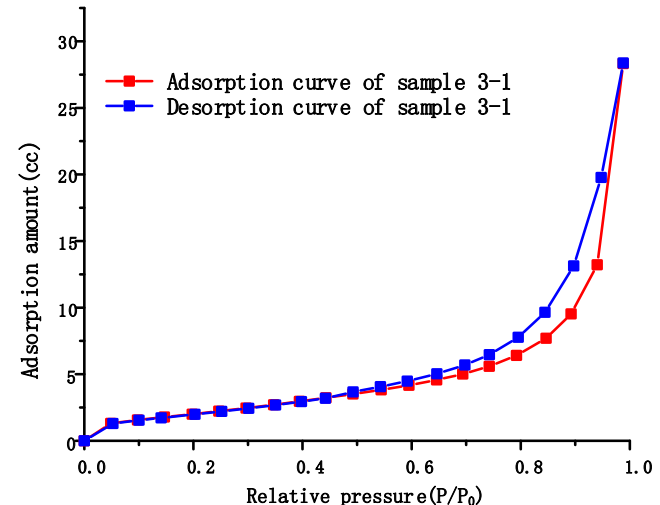

(a) Natural condition

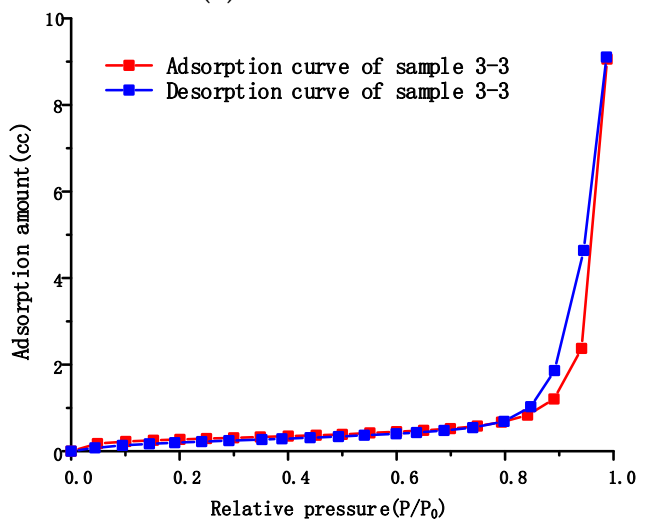

(c) Crude oil erosion

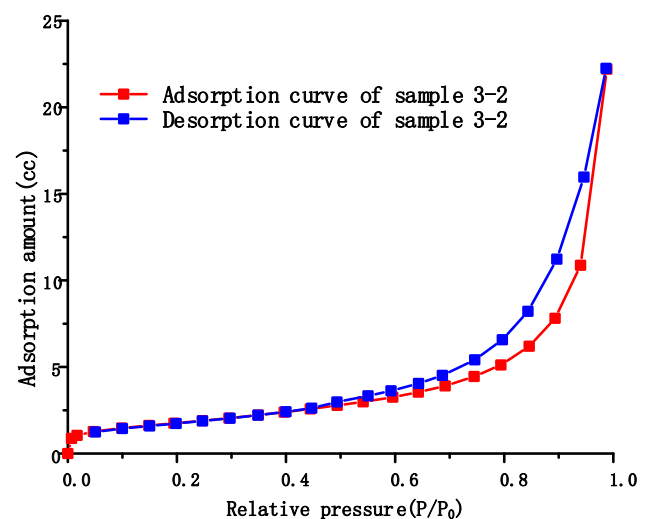

(b) Brine erosion

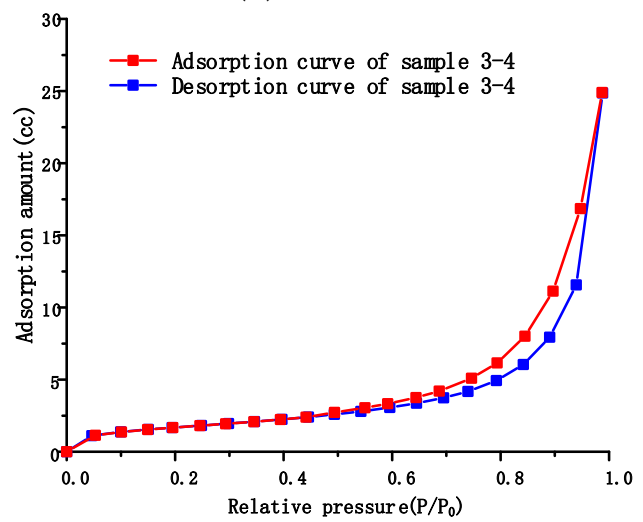

(d) Oil washing

Figure 8. The adsorption isotherms of caprock samples under different sample treatment conditions.

\subsection{Specific Surface Area Variation Characteristics}

The specific surface area refers to the total surface area $\left(\mathrm{m}^{2} / \mathrm{g}\right)$ of a unit mass substance. Gas adsorption method is considered as one of the most authoritative test methods to measure it [44]. There are many theories to calculate the specific surface by gas adsorption, of which the BET theory is most widely used in the calculation of specific surface. In order to obtain the specific surface area by BET plot, the saturated adsorption amount is determined from the slope and intercept of the straight line portion of the plot.

According to the BET equation, the relative pressure $x$ is replaced by $P / P_{0}$

$$
c \frac{P / P_{0}}{v\left(1-P / P_{0}\right)}=\frac{1}{v_{m} C}+\frac{C-1}{v_{m} C} \frac{P}{P_{0}}
$$

where $v$ is the adsorption amount corresponding to the relative pressure $P / P_{0} ; v_{m}$ is the saturated adsorption capacity of monolayer; $C$ is a physical quantity related to the difference between the adsorption heat and condensation heat of the first layer, and $C$ is a constant when the adsorbate, adsorbent and adsorption equilibrium temperature are selected.

It can be seen that when $\left[\left(P / P_{0}\right) /\left[v\left(1-P / P_{0}\right)\right]\right.$ is used to plot $P / P_{0}$, a straight line can be obtained with slope $b=\frac{C-1}{v_{m} \mathrm{C}}$, intercept $a=\frac{1}{v_{m} \mathrm{C}}$, and the saturated adsorption amount of the monolayer can be obtained from slope and intercept.

$$
v_{m}=\frac{1}{a+b}
$$




$$
C=\frac{b}{a}+1
$$

According to the area $a_{m}$ occupied by each adsorbed molecule on the adsorbent surface, the surface area of each gram of solid sample can be calculated. For $\mathrm{N}_{2}$, the area occupied by each nitrogen molecule on the surface of the adsorbent is $16.2 \AA^{2}$, so the area $S_{0}$ occupied by each milliliter of adsorbed $\mathrm{N}_{2}$ molecule when laid into a monolayer should be

$$
S_{0}=\frac{6.023 \times 10^{23} / \mathrm{mol} \times 16.2 \times 10^{-20} \mathrm{~m}^{2}}{22.4 \times 10^{3} \mathrm{~mL} / \mathrm{mol}}=4.36 \mathrm{~m}^{2} / \mathrm{mL}
$$

Therefore, the specific surface area of the sample can be expressed as

$$
c S=S_{0} \cdot v_{m}=4.36 \times v_{m}\left(\mathrm{~m}^{2} / \mathrm{g}\right)
$$

It should be noted that the BET equation only holds in the range of relative pressure $P / P_{0}=$ 0.05 0.35.

BET diagrams of rock salt, interlayer and cap rock under different conditions are shown in Figures 9-11. It can be seen that in the range of relative pressure $P / P_{0}=0.05 \sim 0.35,\left(P / P_{0}\right) /\left[v\left(1-P / P_{0}\right)\right]$ and $P / P_{0}$ have a good linear relationship. According to Equations (2)-(5), the calculated saturated adsorption amount $v_{m}$, constant $C$ and specific surface area $S$ of the single molecular layer of surrounding rocks with different properties under different conditions are shown in Table 7.

According to the BET calculation data in Table 7, the specific surface area of the rock salt sample is the smallest under natural conditions, at only $4.86 \mathrm{~m}^{2} / \mathrm{g}$. The specific surface area of the caprock sample is slightly larger, $8.07 \mathrm{~m}^{2} / \mathrm{g}$. The specific surface area of the interlayer sample is much larger than that of rock salt and caprock, reaching $36.91 \mathrm{~m}^{2} / \mathrm{g}$. Compared with rock salt and cap rock, the porosity in the interlayer is more developed. Although the specific surface areas of rock salt and cap rock are not very different, the reasons for this result are quite different. According to the types of adsorption isotherms of rock salt and cap-rock, it can be judged that the pores of rock salt and cap rock are composed of micropores, mesopores, and macropores. Due to the adsorption isotherms of rock salt samples tending to be saturated when the relative pressure is high, the pore diameters of macropores are all less than $500 \mathrm{~nm}$ (range extremum of the instrument). Moreover, the relatively small pore size has an extremely low specific surface area, which means that the porosity of the rock salt itself is extremely low. On the other hand, the pore size of the caprock sample is relatively larger, and even its macropores are relatively developed. A large number of macropores causes the specific surface area of the cap layer sample to be relatively low.

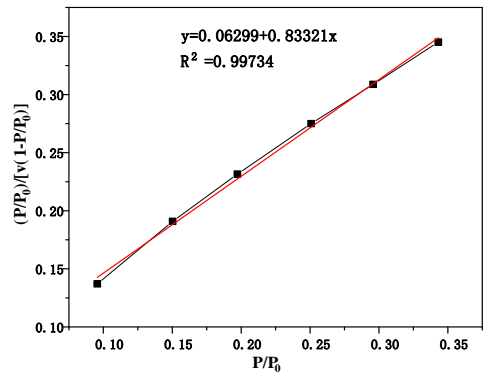

(a) Natural conditio

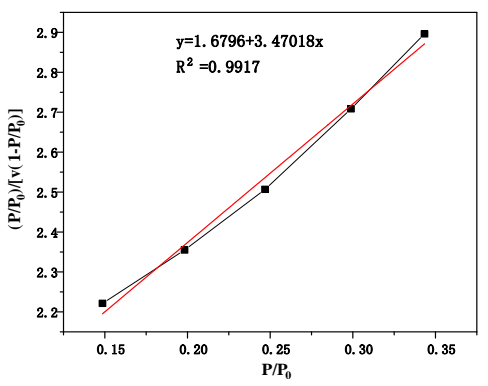

(b) Crude oil erosion

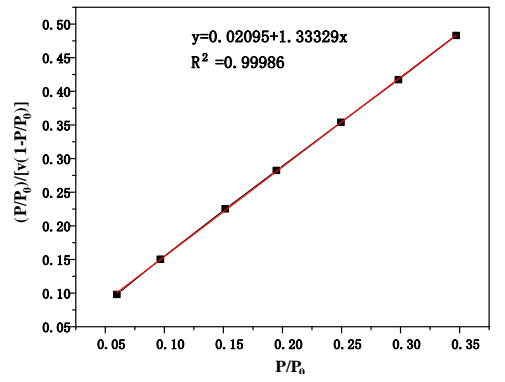

(c) Oil washing

Figure 9. The BET diagrams of rock salt samples after different sample treatment conditions. 


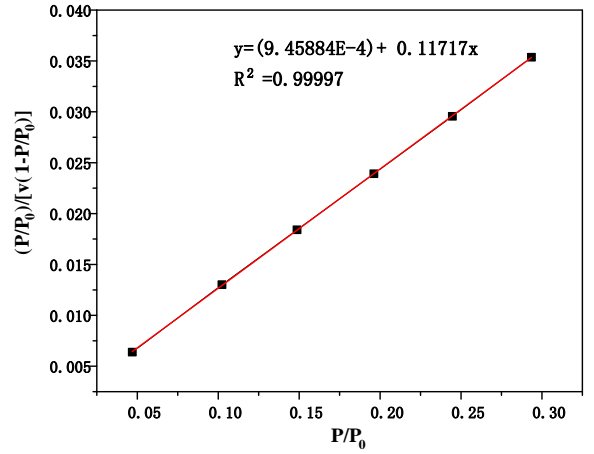

(a) Natural condition

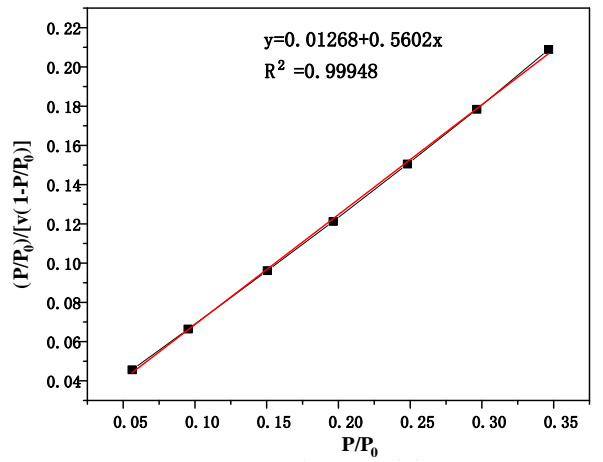

(c) Crude oil erosion

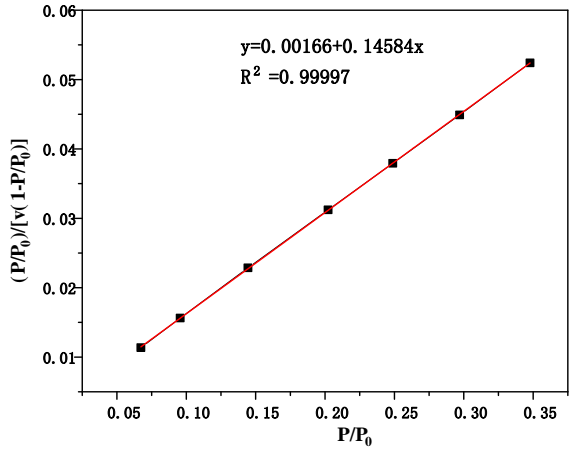

(b) Brine erosion

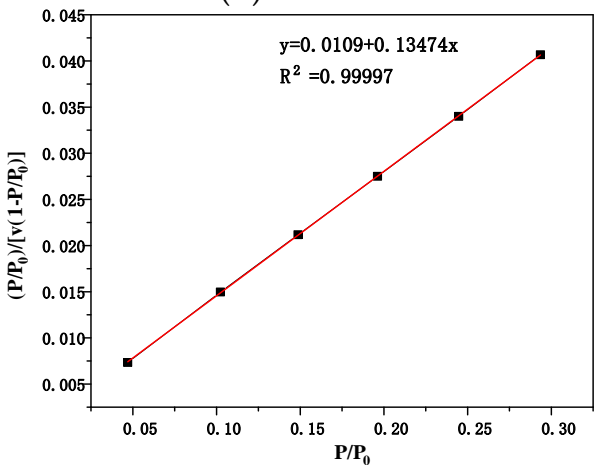

(d) Oil washing

Figure 10. The BET diagrams of interlayer samples after different sample treatment conditions.

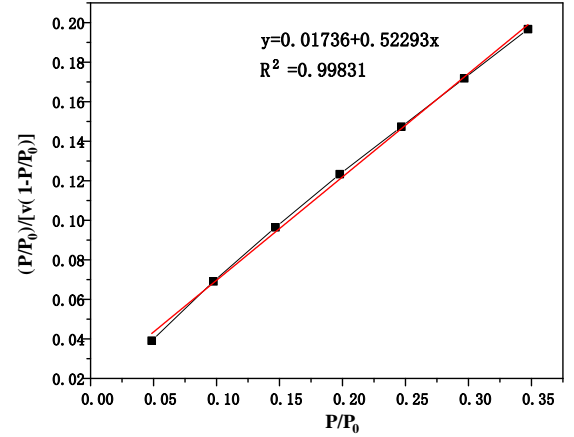

(a) Natural condition

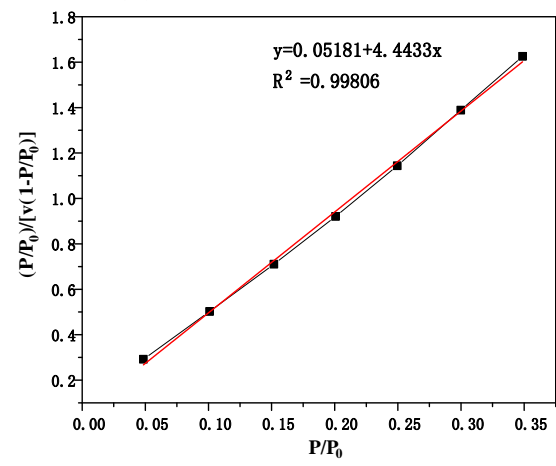

(c) Crude oil erosion

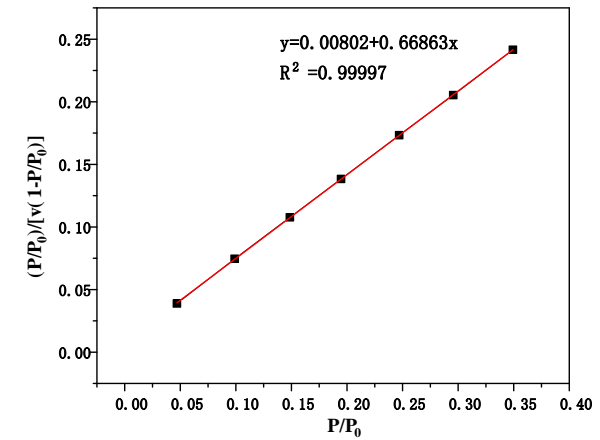

(b) Brine erosion

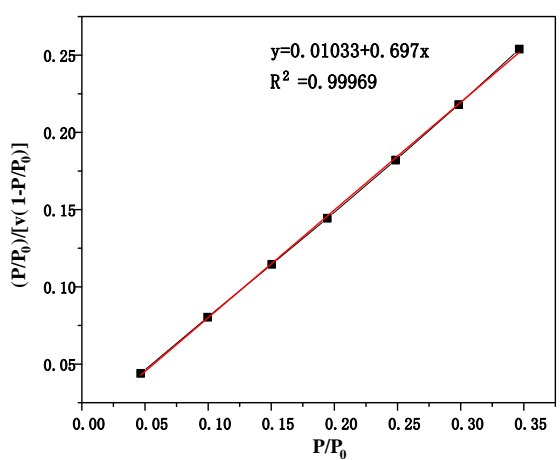

(d) Oil washing

Figure 11. The BET diagrams of cap rock samples after different sample treatment conditions. 
Table 7. The BET data of samples tested after treatment under three different conditions.

\begin{tabular}{ccccccc}
\hline Conditions & Sample & $\boldsymbol{a}(\mathrm{g} / \mathrm{mL})$ & $\boldsymbol{b}(\mathrm{g} / \mathrm{mL})$ & $\boldsymbol{C}$ & $\boldsymbol{v}_{\mathbf{m}}(\mathbf{m L} / \mathbf{g})$ & $S\left(\mathbf{m}^{2} / \mathbf{g}\right)$ \\
\hline Natural environment & $1-1$ & 0.06 & 0.83 & 14.23 & 1.12 & 4.86 \\
Crude oil erosion & $1-2$ & 1.68 & 3.47 & 3.07 & 0.19 & 0.85 \\
Oil washing & $1-3$ & 0.02 & 1.33 & 64.64 & 0.74 & 3.22 \\
Natural environment & $2-1$ & 0.00 & 0.12 & 124.87 & 8.47 & 36.91 \\
Brine erosion & $2-2$ & 0.00 & 0.15 & 88.86 & 6.78 & 29.56 \\
Crude oil erosion & $2-3$ & 0.01 & 0.56 & 45.18 & 1.75 & 7.61 \\
Oil washing & $2-4$ & 0.01 & 0.13 & 13.36 & 6.87 & 29.94 \\
Natural environment & $3-1$ & 0.02 & 0.52 & 31.12 & 1.85 & 8.07 \\
Brine erosion & $3-2$ & 0.01 & 0.67 & 84.37 & 1.48 & 6.44 \\
Crude oil erosion & $3-3$ & 0.05 & 4.44 & 86.76 & 0.22 & 0.97 \\
Oil washing & $3-4$ & 0.01 & 0.70 & 68.47 & 1.41 & 6.16 \\
\hline
\end{tabular}

Under the condition of crude oil erosion, the specific surface area of rock salt, interlayer and caprock samples are greatly reduced compared with those seen under natural conditions. Rock salt sample 1-2 has a specific surface area $S=0.85$, much less than $4.86 \mathrm{~m}^{2} / \mathrm{g}$; the specific surface area of interlayer sample 2-3 is $S=7.61$, much less than $36.91 \mathrm{~m}^{2} / \mathrm{g}$; the specific surface area s of interlayer sample $3-3$ is $S=0.97$, much less than $8.07 \mathrm{~m}^{2} / \mathrm{g}$. It is considered that the reason for these results is the three inferences, as follows:

(1) The residual organic matter in crude oil covers or fills the pores in the sample, causing a temporary decrease in specific surface area, which can be recovered by washing with oil, and crude oil will not damage the sample;

(2) Crude oil erosion causes a large number of pores in the sample to communicate, leading to an increase in the average pore diameter of the sample, and resulting in a permanent decrease in the specific surface area.

(3) Both the filling of pores by organic matter in crude oil and the pore connectivity caused by crude oil erosion cause the specific surface area of the sample to decrease, which can be partially recovered by washing oil.

BET data of samples 1-3, 2-4 and 3-4 after oil washing revealed the potential reason for the large reduction of specific surface area. The specific surface area of rock salt sample 1-3 increases to $3.22 \mathrm{~m}^{2} / \mathrm{g}$ after oil washing, returning to $66.3 \%$ of the natural specific surface area; the specific surface area of interlayer sample 2-4 increased to $29.94 \mathrm{~m}^{2} / \mathrm{g}$, returning to $81.1 \%$ of the natural specific surface area. The specific surface area of the cap layer sample 3-4 increased to $6.16 \mathrm{~m}^{2} / \mathrm{g}$ and recovered to $76.4 \%$ of the natural specific surface area. This shows that the reduction in the specific surface is recoverable to a certain extent, but it cannot be $100 \%$ recovered. Therefore, this result verifies the possibility of deduction (3). However, considering that organic solvent cannot dissolve all the residual organic matter in pores, this result cannot exclude deduction (1). It is necessary to further verify the occurrence of damage during crude oil erosion through mechanical tests. Nonetheless, this further indicates that it is highly possible for the hard-volatile organic matter in crude oil to cover and fill rock pores.

However, the specific surface area of interlayer and caprock decreases under the condition of brine erosion. This is due to the water-rock interaction resulting in some pores of interlayer and cap rock samples being connected, and the dissolution of soluble substances (such as rock salt particles) in the interlayer, and cap-rock also causes the appearance of small pores to become large pores, resulting in the reduction in the specific surface area of the samples. This also shows that, compared with oil-rock interaction, water-rock interaction is more likely to cause irreversible damage to the rock skeleton.

\section{Total Aperture Distribution Characteristics}

The pore size distribution refers to the percentage of pore sizes of all levels in the material in terms of quantity or volume. IUPAC uses the effective average molecular diameter of nitrogen molecules 
(adsorbates) as a benchmark to propose a classification method for pores, i.e., a universal classification: Ultramicropore $\mathrm{r}<0.5 \mathrm{~nm}$; Micropore $0.5 \mathrm{~nm}<\mathrm{r}<2.0 \mathrm{~nm}$; Mesopore $2.0 \mathrm{~nm}<\mathrm{r}<50.0 \mathrm{~nm}$; Macroporous $\mathrm{r}>50.0 \mathrm{~nm}$.

At present, the gas adsorption method and the mercury intrusion method are used to measure the pore size distribution of rocks. Theoretically, the nitrogen adsorption method has higher measurement accuracy only for micropores and mesopores below $50 \mathrm{~nm}$, while the measurement accuracy for larger pores will be greatly reduced. For the mercury intrusion method, although its measurable minimum pore size can reach $3.75 \mathrm{~nm}$, the high-pressure mercury intrusion method has a higher measurement accuracy for large pores above $50 \mathrm{~nm}$ because of the skeleton structure of rock samples will be destroyed with the increase in mercury pressure [45-49]. By combining the gas adsorption method and mercury intrusion measurement range we can obtain the continuous distribution of micropores, mesopores and macropores in the rock surrounding the SPR salt cavern. In this study, the gas adsorption method and mercury intrusion method are combined to obtain the total pore size distribution of the rock.

In the data processing process, the gas adsorption data partially overlapped with the mercury injection data, with the upper limit pore diameter of mesopores being $50 \mathrm{~nm}$ as the boundary. For the pore diameter distribution in the 0-50 $\mathrm{nm}$ micro-mesopore range, we use the gas adsorption data, and for the pore diameter distribution in the larger than $50 \mathrm{~nm}$ range, the mercury injection method. The data are connected at the $50 \mathrm{~nm}$ boundary to obtain the total pore diameter distribution map of each test sample.

\subsection{Total Pore Diameter Distribution of Rock Salt Samples}

The total pore size distribution characteristics of rock salt samples under different conditions are shown in Figure 12. Under natural conditions, the pore diameter of rock salt is mostly in the range of $3.4 \sim 50 \mathrm{~nm}$ and greater than $29.6 \mu \mathrm{m}$. The pore diameter distribution of rock salt in the larger pore range is not uniform. However, according to the scanning results of the electron microscope of the rock salt sample, its pore size is much smaller than $29.6 \mu \mathrm{m}$, so it can be considered that the pore size larger than $29.6 \mu \mathrm{m}$ in the mercury injection results of the rock salt sample is more likely to be caused by the micro-cracks in the crystal grains of the rock salt.

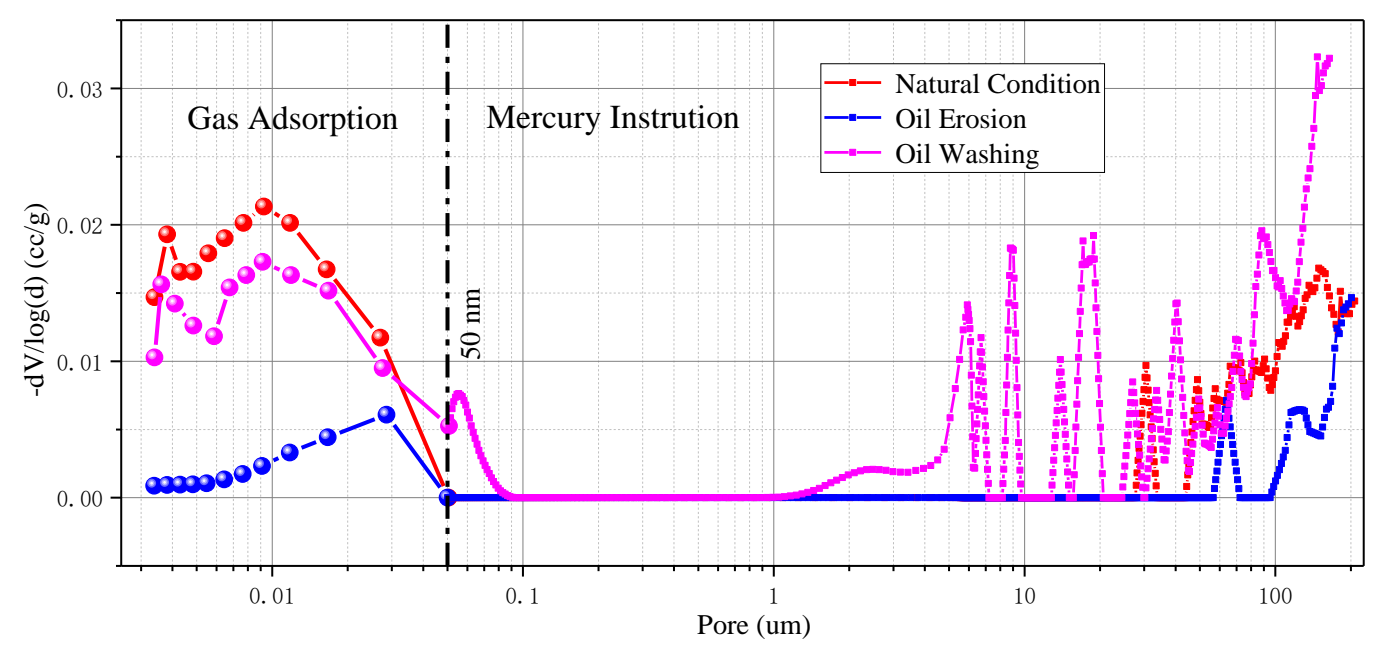

Figure 12. The total pore size distribution of rock salt samples under different conditions.

Under the influence of crude oil, the pore volume corresponding to each pore diameter of rock salt is obviously reduced. The pore volume of some large pores is even directly reduced to 0 under the influence of crude oil, which is still caused by the blockage of pores by nonvolatile organic matter in crude oil. However, the pore size distribution of rock salt after washing with oil is obviously different from that under natural conditions. Although the pore size distribution curve after oil washing is close to that of natural conditions in the mesoporous range, the distribution of macropores is quite different. 
Not only is the pore size range obviously increased, but the pore size distribution has a large fluctuation. This is caused by the $1.01 \%$ water content in crude oil. Even though the water content in crude oil is extremely low, rock salt is extremely sensitive to fresh water and dissolves in it. Therefore, a small amount of water intrusion will damage the pore structure of rock salt samples, causing the secondary development of pores near micro-fissures in the rock salt to increase the pore size distribution range of large pores. However, this has little effect on the distribution of micro-mesopores in rock salt.

\subsection{Total Pore Diameter Distribution of Interlayer Samples}

The total pore size distribution characteristics of interlayers samples under different conditions are shown in Figure 13. The pore size distribution of interlayers samples has two significant peaks under natural conditions, which are respectively distributed between $3.4 \sim 62.5 \mathrm{~nm}$ and $43.7 \sim 60.9 \mu \mathrm{m}$. It is considered that the pore size range of $3.4 \sim 62.5 \mathrm{~nm}$ is closer to the natural pore structure distribution of the interlayer sample, while the pore size of $43.7 \sim 60.9 \mu \mathrm{m}$ can be considered as the micro-crack spacing range in the interlayer sample.

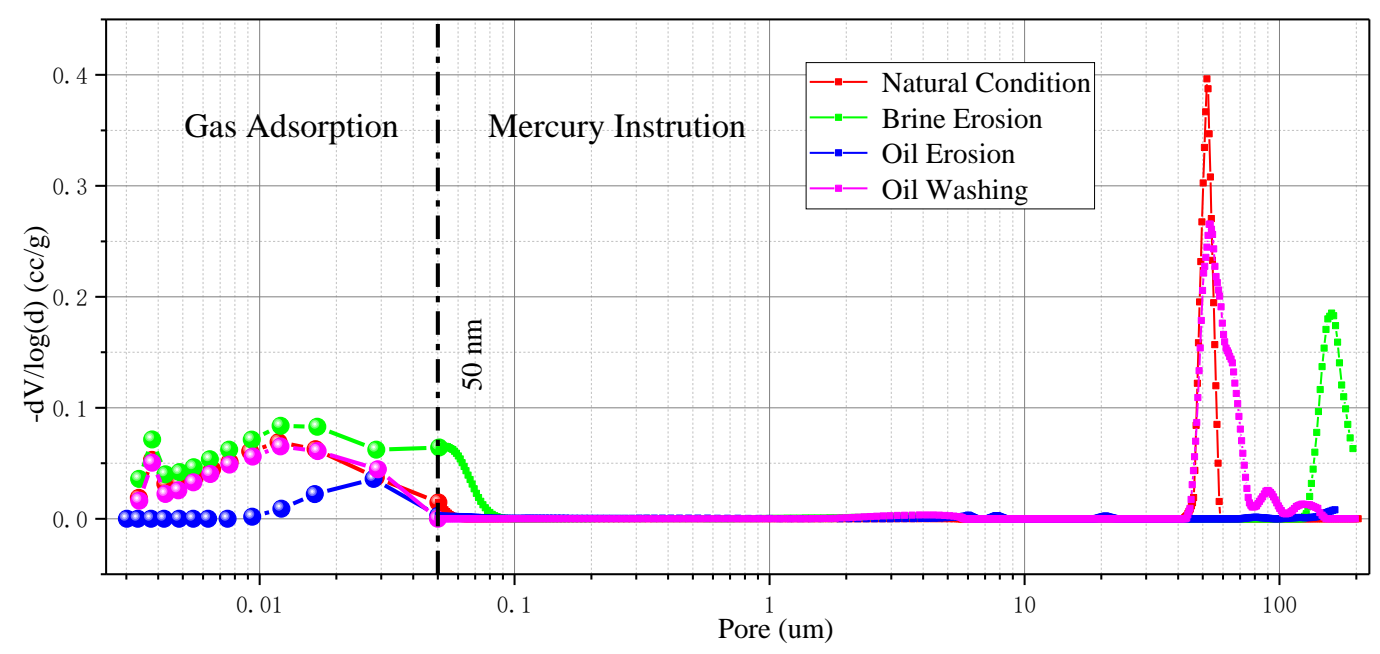

Figure 13. The total pore size distribution of interlayer samples under different conditions.

Under the condition of crude oil erosion, like the pore size distribution characteristics of rock salt samples, a large number of pores are blocked, resulting in obvious changes in the pore structure of interlayer samples. The pore volume corresponding to the pore diameter is also significantly reduced due to the blockage of pores. The pore size distribution curve near the mesopores after oil washing is basically the same as that under natural conditions, and the pore sizes corresponding to the two peaks of the curve are completely the same. However, the pore size distribution range of macropores after oil washing is slightly larger than that under natural conditions, which may be caused by slight water-rock interaction or slight structural damage caused by oil-rock interaction due to extremely low water content in crude oil, but we cannot exclude the possibility that the slight difference is caused by heterogeneity of the sample itself.

However, brine erosion obviously changed the pore size distribution of interlayer samples. The distribution curve of both nano-sized pores and micro-sized fissures shows an obvious shift to the right. This means that a large amount of water-rock interaction increases the pore sizes of interlayer samples and changes their pore structure characteristics.

\subsection{Total Pore Diameter Distribution of Caprock Samples}

The total pore size distribution characteristics of caprock samples under different conditions are shown in Figure 14. Different from salt rock and interlayer, the total pore size distribution curve of cap rock samples under natural conditions has only one peak, and its pore size is widely distributed in the 
range of $3.0 \sim 75.9 \mathrm{~nm}$. This shows that the pore size distribution of the caprock is relatively uniform and there are few microcracks in the caprock. This is beneficial to the sealing performance of salt cavern storage.

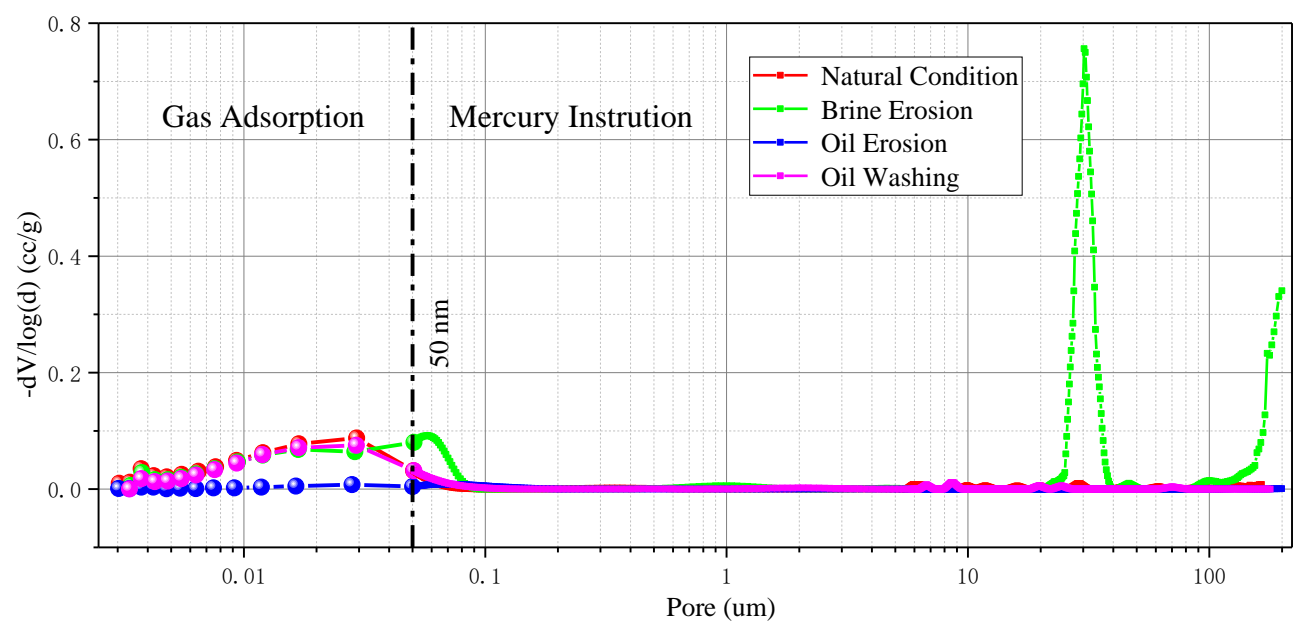

Figure 14. The total pore size distribution of cap rock samples under different conditions.

Similar to the pore size distribution characteristics of interlayer samples under crude oil erosion conditions, the blockage of organic matter in crude oil results in the change in the pore size distribution and the decrease in the pore volume of caprock samples under crude oil erosion conditions. However, the pore size distribution curve of the cap layer sample after oil washing basically coincides with the distribution curve under natural conditions but slightly fluctuates between 8 and $100 \mu \mathrm{m}$. This shows that crude oil erosion does not significantly change the structural characteristics of nano-scale pores in caprock samples but has a slight promoting effect on micro-scale micro-fractures. The pore size distribution of caprock samples under brine erosion has obviously changed compared with natural conditions. The generation of a large number of water-rock reactions increases the pore size of caprock and changes its pore structure.

Comparing Figures 12-14, although the nano-scale pore size distribution range between rock salt and interlayer and caprock samples is not very different, the pore volume corresponding to the same pore size differs significantly, which means that the porosity of rock salt is far lower than that of interlayer and caprock. Significantly, compared with natural conditions, the curves of interlayer and caprock samples after oil-washing show a basically consistent distribution of nano-sized pores and a slightly different distribution of micron-sized pores, and both show that crude oil erosion slightly promotes the development of macro-pores. It can be concluded that this phenomenon is not only caused by the heterogeneity of the sample itself, but also due to oil-rock interaction or slight water-rock interaction.

\section{Discussion}

The core of changes in the pore characteristics of surrounding rock is the plugging effect of colloid and asphaltene in crude oil on rock pores and the erosion effect of brine. Although the above test results have verified this assumption, it is still only a reasonable inference based on test data. In order to directly characterize these phenomena, the samples under different erosion environments of interlayer were scanned by an electron microscope. The results are shown in Figure 15. 


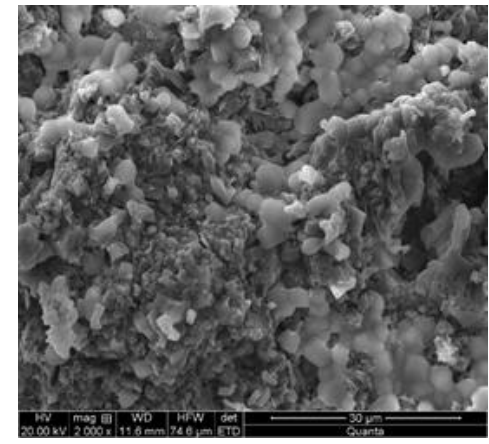

(a) Natural condition

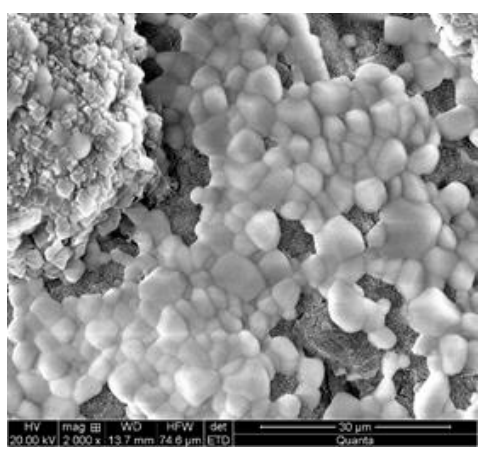

(b) Crude oil erosion

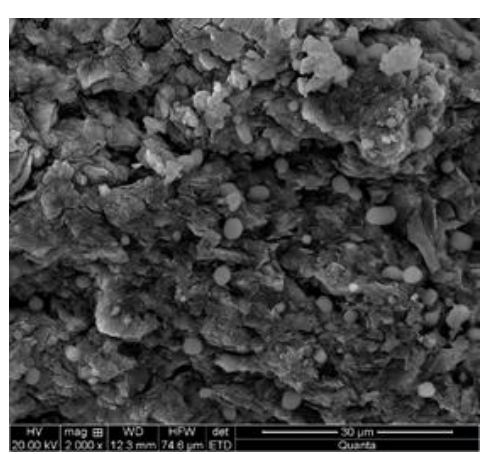

(c) Brine erosion

Figure 15. The SEM result of the interlayer samples under different immersion conditions.

When the interlayer is eroded by crude oil, compared with the scanning results of electron microscopy under conventional conditions, the pores and microcracks on the surface of the sample are covered by a layer of medium, which directly confirms the assumption of the pore blockage being caused by macromolecular organic matter (colloid and asphaltene) in crude oil. However, it can be seen from the interface of the interlayer sample under brine erosion that a large number of microcracks appear at the interface due to brine erosion, the cementation between clay minerals and grains loosens, and a large number of grains and clay are dissolved and transported by brine, resulting in a significant increase in interlayer pores.

\section{Summary and Conclusions}

(1) The surface of the rock salt sample is extremely dense, the crystal grains are large and the cementation between the crystal grains is extremely tight, which reveals the essential reason for the extremely low permeability and porosity of the salt rock. Compared with salt rock, a large number of voids in interlayer and caprock may provide favorable channels for leakage of high-pressure crude oil and brine;

(2) Under the condition of crude oil erosion, the changes in the specific surface area and pore size distribution characteristics of salt rock, interlayer and cap rock samples are caused by the blockage of pores by macromolecular organic matter (colloid and asphaltene) in crude oil, which will not significantly change the structural characteristics of the rock skeleton, which is beneficial to the long-term operation of the SPR salt cavern;

(3) Under brine erosion, the change in the specific surface area and pore size distribution characteristics of interlayer and caprock samples is caused by the dissolution and migration of a large number of crystal grains and clay by brine due to water-rock interaction, leading to the observation that some micro-pores of interlayer and caprock samples turn into macro-pores. This brings obvious changes in the pore structure of the rock and increases the leakage risk of the storage medium. It is suggested that the circulation time of brine in the SPR salt cavern should be reduced as much as possible after the completion of cavern construction.

Author Contributions: Conceptualization, N.Z. and X.S.; Methodology, Y.Z.; Investigation, N.Z. and W.L.; Writing — original draft preparation, N.Z. and P.S.; writing—review and editing, X.S. and P.S. All authors have read and agreed to the published version of the manuscript.

Funding: The authors wish to acknowledge the financial supports of State key laboratory of geotechnical mechanics and engineering open fund (Z019012), the National Natural Science Foundation of China (No. 51904227, 51774266), the 973 Key National Basic Research Program of China (No. 2015CB251602), the Key Natural Science Foundation of Shaanxi Province (No. 2019JLZ-04), Chongqing Basic Research and Frontier Exploration Project (cstc2018jcyjAX0441).

Acknowledgments: The authors are sincerely grateful to J.J.K Daemen (Mackay School of Earth Sciences and Engineering, University of Nevada), for his thoughtful review of this paper. 
Conflicts of Interest: The authors declare no conflict of interest.

\section{References}

1. Brodny, J.; Tutak, M. Analyzing Similarities between the European Union Countries in Terms of the Structure and Volume of Energy Production from Renewable Energy Sources. Energies 2020, 13, 913. [CrossRef]

2. Tutak, M.; Brodny, J. Forecasting Methane Emissions from Hard Coal Mines Including the Methane Drainage Process. Energies 2019, 12, 3840. [CrossRef]

3. Karthikeyan, K.; Sunder, R.; Shankar, K.; Lakshmanaprabu, S.K.; Vijayakumar, V.; Elhoseny, M.; Manogaran, G. Energy consumption analysis of Virtual Machine migration in cloud using hybrid swarm optimization (ABC-BA). J. Supercomput. 2018, 5, 1-17. [CrossRef]

4. Qiao, W.; Tian, W.; Tian, Y.; Yang, Q.; Wang, Y.; Zhang, J. The forecasting of PM2. 5 using a hybrid model based on wavelet transform and an improved deep learning algorithm. IEEE Access 2019, 7, 142814-142825. [CrossRef]

5. Ruiz, L.G.B.; Rueda, R.; Cuéllar, M.P.; Pegalajar, M.C. Energy consumption forecasting based on Elman neural networks with evolutive optimization. Expert Syst. Appl. 2018, 92, 380-389. [CrossRef]

6. Chunrong Tian. Oil Import and Export in China. China Oil Gas 2016, 23, 36-42.

7. Qiao, W.; Yang, Z. Forecast the electricity price of US using a wavelet transform-based hybrid model. Energy 2020, 193, 116704. [CrossRef]

8. Wu, G.; Fan, Y.; Liu, L.C.; Wei, Y.M. An empirical analysis of the dynamic programming model of stockpile acquisition strategies for China's strategic petroleum reserve. Energy Policy 2008, 36, 1470-1478. [CrossRef]

9. Qiao, W.; Yang, Z.; Kang, Z.; Pan, Z. Short-term natural gas consumption prediction based on Volterra adaptive filter and improved whale optimization algorithm. Eng. Appl. Artif. Intell. 2020, 87, 103323. [CrossRef]

10. Chen, J.; Peng, H.; Fan, J.; Zhang, X.; Liu, W.; Jiang, D. Microscopic investigations on the healing and softening of damaged salt by uniaxial deformation from CT, SEM and NMR: Effect of fluids (brine and oil). RSC Adv. 2020, 10, 2877-2886. [CrossRef]

11. Qiao, W.; Huang, K.; Azimi, M.; Han, S. A novel hybrid prediction model for hourly gas consumption in supply side based on improved whale optimization algorithm and relevance vector machine. IEEE Access 2019, 7, 88218-88230. [CrossRef]

12. Singh, S.; Yassine, A. Big data mining of energy time series for behavioral analytics and energy consumption forecasting. Energies 2018, 11, 452. [CrossRef]

13. Weibiao, Q.; Bingfan, L.; Zhangyang, K. Differential scanning calorimetry and electrochemical tests for the analysis of delamination of 3PE coatings. Int. J. Electrochem. Sci. 2019, 14, 7389-7400. [CrossRef]

14. Zhang, N.; Shi, X.; Zhang, Y.; Shan, P. Tightness Analysis of Underground Natural Gas and Oil Storage Caverns with Limit Pillar Widths in Bedded Rock Salt. IEEE Access 2020, 8, 12130-12145. [CrossRef]

15. Zhao, C.; Chen, B. China's oil security from the supply chain perspective: A review. Appl. Energy 2014, 136, 269-279. [CrossRef]

16. Liu, W.; Zhang, X.; Fan, J.Y.; Li, Y.P.; Wang, L. Potential evaluation of salt cavern gas storage in the Yangtze River Delta region and integration of brine extraction-cavern utilization. Nat. Resour. Res. 2020, 3, 1-16. [CrossRef]

17. Li, J.; Tang, Y.; Shi, X.; Xu, W.; Yang, C. Modeling the construction of energy storage salt caverns in bedded salt. Appl. Energy 2019, 255, 113866. [CrossRef]

18. Jinlong, L.; Wenjie, X.; Jianjing, Z.; Wei, L.; Xilin, S.; Chunhe, Y. Modeling the mining of energy storage salt caverns using a structural dynamic mesh. Energy 2020, 193, 116730. [CrossRef]

19. Liu, W.; Zhang, Z.; Chen, J.; Fan, J.; Jiang, D.; Jjk, D.; Li, Y. Physical simulation of construction and control of two butted-well horizontal cavern energy storage using large molded rock salt specimens. Energy 2019, 185, 682-694. [CrossRef]

20. Long-Term Strategic Review of the U.S. Strategic Petroleum Reserve Report to Congress; United States Department of Energy: Washington, DC, USA, 2016.

21. Wu, G.; Wei, Y.M.; Nielsen, C.; Lu, X.; McElroy, M.B. A dynamic programming model of China's strategic petroleum reserve: General strategy and the effect of emergencies. Energy Econ. 2012, 34, 1234-1243. [CrossRef] 
22. Zhang, N.; Shi, X.; Wang, T.; Yang, C.; Liu, W.; Ma, H.; Daemen, J.J.K. Stability and availability evaluation of underground strategic petroleum reserve (SPR) caverns in bedded rock salt of Jintan, China. Energy 2017, 134, 504-514. [CrossRef]

23. Fan, J.; Jiang, D.; Liu, W.; Wu, F.; Chen, J.; Daemen, J.J.K. Discontinuous fatigue of salt rock with low-stress intervals. Int. J. Rock Mech. Min. Sci. 2019, 115, 77-86. [CrossRef]

24. Chen, J.; Lu, D.; Liu, W.; Fan, J.; Jiang, D.; Yi, L.; Kang, Y. Stability study and optimization design of small-spacing two-well (SSTW) salt caverns for natural gas storages. J. Energy Storage 2020, 27, 101131. [CrossRef]

25. Liu, W.; Li, Y.P.; Yang, C.H.; Ma, H.L.; Liu, J.X.; Wang, B.W.; Huang, X.L. Investigation on permeable characteristics and tightness evaluation of typical interlayers of energy storage caverns in bedded salt rock formations. Chin. J. Rock Mech. Eng. 2014, 33, 500-506.

26. Liu, W.; Zhang, Z.X.; Fan, J.Y.; Jiang, D.Y.; Daemen, J.J.K. Research on the stability and treatments of natural gas storage caverns with different shapes in bedded salt rocks. IEEE Access 2020, 000507. [CrossRef]

27. Liu, W.; Zhang, Z.; Chen, J.; Jiang, D.; Wu, F.; Fan, J.; Li, Y. Feasibility evaluation of large-scale underground hydrogen storage in bedded salt rocks of China: A case study in Jiangsu province. Energy 2020. [CrossRef]

28. Zhang, N.; Ma, L.; Wang, M.; Zhang, Q.; Li, J.; Fan, P. Comprehensive risk evaluation of underground energy storage caverns in bedded rock salt. J. Loss Prev. Process Ind. 2017, 45, 264-276. [CrossRef]

29. Feng, C.; Zhaoyuan, L.; Jianqiang, C.; Bo, C.; Yanbin, Y.; Changlu, L.; Chong, J. Research on Reducing Mining-Induced Disasters by Filling in Steeply Inclined Thick Coal Seams. Sustainability 2019, 11, 5802. [CrossRef]

30. Cui, F.; Yang, Y.; Lai, X.; Jia, C.; Shan, P. Experimental Study on the Effect of Advancing Speed and Stoping Time on the Energy Release of Overburden in an Upward Mining Coal Working Face with a Hard Roof. Sustainability 2020, 12, 37. [CrossRef]

31. Fan, J.; Xie, H.; Chen, J.; Jiang, D.; Li, C.; Tiedeu, W.N.; Ambre, J. Preliminary feasibility analysis of a hybrid pumped-hydro energy storage system using abandoned coal mine goafs. Appl. Energy 2020, 258, 114007. [CrossRef]

32. Shan, P.H.; Lai, X.P.; Liu, X.M. An associated evaluation methodology of initial stress level of coal-rock masses in steeply inclined coal seams, Urumchi coal field, China. Eng. Comput. 2020. [CrossRef]

33. Zhang, N.; Yang, C.; Shi, X.; Wang, T.; Yin, H.; Daemen, J.J.K. Analysis of mechanical and permeability properties of mudstone interlayers around a strategic petroleum reserve cavern in bedded rock salt. Int. J. Rock Mech. Min. Sci. 2018, 112, 1-10. [CrossRef]

34. Chen, J.; Li, E.; Luo, J. Characterization of Microscopic Pore Structures of Rock Salt through Mercury Injection and Nitrogen Absorption Tests. Geofluids 2018, 2018, 1-7. [CrossRef]

35. De Las Cuevas, C. Pore structure characterization in rock salt. Eng. Geol. 1997, 47, 17-30. [CrossRef]

36. Li, Y.P.; Yang, C.H. Three-dimensional expanded Cosserat medium constitutive model for laminated salt rock. Rock Soil Mech. 2006, 27, 509-513.

37. Shi, X.L.; Li, Y.P.; Yang, C.H.; Qu, D.A. Test study of influence of brine on tensile strength of muddy intercalation. Chin. J. Rock Mech. Eng. 2009, 28, 2301-2308.

38. Wei, L.; Jie, C.; Deyi, J.; Xilin, S.; Yinping, L.; Daemen, J.J.K.; Chunhe, Y. Tightness and suitability evaluation of abandoned salt caverns served as hydrocarbon energies storage under adverse geological conditions (AGC). Appl. Energy 2016, 178, 703-720. [CrossRef]

39. Yang, Y.; Aplin, A.C. A permeability-porosity relationship for mudstones. Mar. Pet. Geol. 2010, 27, $1692-1697$. [CrossRef]

40. Ji, W.; Yang, C.H.; Liu, W.; Li, M. Experimental investigation on meso-pore structure properties of bedded salt rock. Chin. J. Rock Mech. Eng. 2013, 32, 2036-2044.

41. Li, J.; Shi, X.; Zhang, S. Construction modeling and parameter optimization of multi-step horizontal energy storage salt caverns. Energy 2020, 190, 107058.

42. Yan, J.; Zhang, Q. Adsorption and Condensation; Science Press: Beijing, China, 1976.

43. Miyata, T.; Endo, A.; Ohmori, T.; Akiya, T.; Nakaiwa, M. Evaluation of pore size distribution in boundary region of micropore and mesopore using gas adsorption method. J. Colloid Interface Sci. 2003, 262, 116-125. [CrossRef]

44. Abdel-Jawad, Y.; Hansen, W. Pore Structure of Hydrated Cement Determined By Mercury Porosimetry And Nitrogen Sorption Techniques. MRS Proc. 1988, 137, 105. [CrossRef] 
45. Qiuquan, C.; Hongyu, C.; Ming, F.; Qiang, W.; Weijun, C. Determination of the total pore texture of caprock. Pet. Geol. Exp. 2006, 28, 604 .

46. Qiao, W.; Yang, Z. An improved dolphin swarm algorithm based on Kernel Fuzzy C-means in the application of solving the optimal problems of large-scale function. IEEE Access 2019, 8, 2073-2089. [CrossRef]

47. Qiao, W.; Yang, Z. Solving large-scale function optimization problem by using a new metaheuristic algorithm based on quantum dolphin swarm algorithm. IEEE Access 2019, 7, 138972-138989. [CrossRef]

48. Qiao, W.; Yang, Z. Modified dolphin swarm algorithm based on chaotic maps for solving high-dimensional function optimization problems. IEEE Access 2019, 7, 110472-110486. [CrossRef]

49. Zhang, Y.; Cao, S.G.; Zhang, N.; Zhao, C.Z. The application of short-wall block backfill mining to preserve surface water resources in northwest China. J. Clean. Prod. 2020. [CrossRef]

(C) 2020 by the authors. Licensee MDPI, Basel, Switzerland. This article is an open access article distributed under the terms and conditions of the Creative Commons Attribution (CC BY) license (http://creativecommons.org/licenses/by/4.0/). 